\title{
A FINITE ELEMENT METHOD FOR THE SURFACE STOKES PROBLEM
}

\author{
MAXIM A. OLSHANSKII*, ANNALISA QUAINI ${ }^{\dagger}$, ARNOLD REUSKEN ${ }^{\ddagger}$, AND VLADIMIR \\ YUSHUTIN §
}

\begin{abstract}
We consider a Stokes problem posed on a 2D surface embedded in a 3D domain. The equations describe an equilibrium, area-preserving tangential flow of a viscous surface fluid and serve as a model problem in the dynamics of material interfaces. In this paper, we develop and analyze a Trace finite element method (TraceFEM) for such a surface Stokes problem. TraceFEM relies on finite element spaces defined on a fixed, surface-independent background mesh which consists of shape-regular tetrahedra. Thus, there is no need for surface parametrization or surface fitting with the mesh. The TraceFEM treated here is based on $P_{1}$ bulk finite elements for both the velocity and the pressure. In order to enforce the velocity vector field to be tangential to the surface we introduce a penalty term. The method is straightforward to implement and has an $O\left(h^{2}\right)$ geometric consistency error, which is of the same order as the approximation error due to the $P_{1}-P_{1}$ pair for velocity and pressure. We prove stability and optimal order discretization error bounds in the surface $H^{1}$ and $L^{2}$ norms. A series of numerical experiments is presented to illustrate certain features of the proposed TraceFEM.
\end{abstract}

Key words. Surface fluid equations; Surface Stokes problem; Trace finite element method.

1. Introduction. Fluid equations on manifolds appear in the literature on modeling of emulsions, foams and biological membranes. See, for example, 40, 41, 3, 5, 34, 33. They are also studied as an interesting mathematical problem in its own right in, e.g., [12, 43, 42, 2, 27, 1, 25. Despite the apparent practical and mathematical relevance, so far fluids on manifolds have received little attention from the scientific computing community. Only few papers, like for instance [23, 28, 4, 37, 36, 38, 15], treat the development and analysis of numerical methods for surface fluid equations or coupled bulk-surface fluid problems. Among those papers, [38, 15] applied surface finite element methods to discretize the incompressible surface Navier-Stokes equations in primitive variables on stationary manifolds. In [38, the authors considered $P_{1}-P_{1}$ finite elements with no pressure stabilization and a penalty technique to force the flow field to be tangential to the surface. In [15, instead, surface Taylor-Hood elements are used and combined with a Lagrange multiplier method to enforce the tangentiality constraint. Neither references address the numerical analysis of finite element methods for the surface Navier-Stokes equations. In general, we are not aware of any paper containing a rigorous analysis of finite element (or any other) discretization methods for surface (Navier-)Stokes equations.

In recent years, several papers on discretization methods for scalar elliptic and parabolic partial differential equations on surfaces have appeared. We refer to [11, 31] for a review on surface finite element methods. Only very recently finite element methods have been applied and analyzed for vector Laplace equations on surfaces [22, 17.

*Department of Mathematics, University of Houston, Houston, Texas 77204 (molshan@math.uh.edu); Partially supported by NSF through the Division of Mathematical Sciences grants 1522252 and 1717516.

${ }^{\dagger}$ Department of Mathematics, University of Houston, Houston, Texas 77204 (quaini@math.uh.edu); Partially supported by NSF through grant DMS-1263572 and DMS1620384 .,

${ }^{\ddagger}$ Institut für Geometrie und Praktische Mathematik, RWTH-Aachen University, D-52056 Aachen, Germany (reusken@igpm.rwth-aachen.de).

$\S$ Department of Mathematics, University of Houston, Houston, Texas 77204 (yushutin@math.uh.edu) 
This is a first natural step in extending the methods and analyses proposed for the scalar problems to surface (Navier-)Stokes equations. In [22], the authors analyzed a surface FEM combined with a penalty technique to impose the tangentiality constraint. The results include stability and error analysis, which also account for the effects of geometric errors. The approach presented in [17] is different: an unfitted finite element method (TraceFEM) combined with a Lagrange multiplier technique to enforce the discrete vector fields to be (approximately) tangential to the surface. Stability and optimal order error estimates were also proved in 17. The present paper continues along this latter line of research and studies the TraceFEM applied to the Stokes equations posed on a stationary closed smooth surface $\Gamma$ embedded in $\mathbb{R}^{3}$.

The choice of the geometrically unfitted discretization (instead of the surface FEM as in [38, 15]) is motivated by our ultimate goal: the numerical simulation of fluid flows on evolving surfaces $\Gamma(t)[3,25,24$, including cases where a parametrization of $\Gamma(t)$ is not explicitly available and $\Gamma(t)$ may undergo large deformations or even topological changes. Unfitted discretizations, such as TraceFEM, allow to avoid mesh reconstruction for the time-dependent geometry and to treat implicitly defined surfaces. As illustrated in [32, 26], TraceFEM works very well for scalar PDEs posed on evolving surfaces and can be naturally combined with the level set method for (implicit) surface representation. In [24, it is shown that the surface (Navier-)Stokes equations used to model incompressible surface fluid systems on evolving surfaces admit a natural splitting into coupled equations for tangential and normal motions. Such splitting and time discretization yield a subproblem that is very similar to the Stokes problem treated in this paper. Hence, we consider the detailed study of a trace FEM for the Stokes problem on a stationary surface to be an important step in the development of a robust and efficient finite element solver for the Navier-Stokes equations on evolving surfaces. In addition, the method treated in this paper can be used to study interesting properties of Stokes problems on stationary surfaces, as illustrated by the numerical experiments presented in section 7 .

The TraceFEM considered in this paper is based on the $P_{1}-P_{1}$ finite element pair defined on the background mesh. Pressure stabilization is achieved through the simple Brezzi-Pitkäranta stabilization. Unlike [17, we consider a penalty technique for the tangentiality condition. Altogether, this results in a straightforward to use solver for fluid equations posed on surfaces. An alternative approach with higher order (generalized) Taylor-Hood elements and a Lagrange multiplier method will be subject of future work.

The principal contributions of the paper are the following:

- Introduction of an easy to implement TraceFEM, which is based on a piecewise planar approximation of the surface, $P_{1}-P_{1}$ finite elements on the background mesh, Brezzi-Pitkäranta and so-called "volume normal derivative" stabilization terms, and a penalty method for the tangentiality constraint.

- Error analysis showing optimal order $O(h)$ error estimates in the $H^{1}(\Gamma)^{3}$ norm for velocity and $L^{2}(\Gamma)$ norm for pressure, and an optimal $O\left(h^{2}\right)$ error estimate for velocity in the $L^{2}(\Gamma)^{3}$ norm. All these estimates do not depend on the position of $\Gamma$ in the background mesh. The analysis does not account for the effect of geometric errors.

- Study of the conditioning of the resulting saddle point stiffness matrix. We prove that the spectral condition number of this matrix is bounded by $\mathrm{ch}^{-2}$, with a constant $c$ that is independent of the position of the surface $\Gamma$ relative 
to the underlying triangulation.

- Presentation of an optimal preconditioner.

The outline of the paper is as follows. In section 2, we introduce the surface Stokes system and some notions of tangential differential calculus. We give a weak formulation of the problem and recall some known results. In section 3 , we study the augmented surface Stokes problem, i.e. the problem with additional penalty terms. An estimate on the difference between weak solutions of the original and augmented problem is derived. An unfitted finite element method (TraceFEM) for the surface Stokes problem is introduced in section 4 . The role of the different stabilization terms is explained. In section 5 an error analysis of this method is presented. A discrete infsup stability and optimal a-priori discretization error bounds are proved. In section 6 . we analyze the conditioning properties of the resulting saddle point matrix and an optimal preconditioner is introduced. Numerical results in section 7 illustrate the performance of the method in terms of error convergence, efficiency of the linear solver, and flexibility in handling implicitly defined geometries.

2. Continuous problem. Assume that $\Gamma$ is a closed sufficiently smooth surface in $\mathbb{R}^{3}$. The outward pointing unit normal on $\Gamma$ is denoted by $\mathbf{n}$, and the orthogonal projection on the tangential plane is given by $\mathbf{P}=\mathbf{P}(\mathbf{x}):=\mathbf{I}-\mathbf{n}(\mathbf{x}) \mathbf{n}(\mathbf{x})^{T}, \mathbf{x} \in \Gamma$. In a neighborhood $\mathcal{O}(\Gamma)$ of $\Gamma$ the closest point projection $\mathbf{p}: \mathcal{O}(\Gamma) \rightarrow \Gamma$ is well defined. For a scalar function $p: \Gamma \rightarrow \mathbb{R}$ or a vector function $\mathbf{u}: \Gamma \rightarrow \mathbb{R}^{3}$ we define $p^{e}=p \circ \mathbf{p}: \mathcal{O}(\Gamma) \rightarrow \mathbb{R}, \mathbf{u}^{e}=\mathbf{u} \circ \mathbf{p}: \mathcal{O}(\Gamma) \rightarrow \mathbb{R}^{3}$, extensions of $p$ and $\mathbf{u}$ from $\Gamma$ to its neighborhood $\mathcal{O}(\Gamma)$ along the normal directions. On $\Gamma$ it holds $\nabla p^{e}=\mathbf{P} \nabla p^{e}$ and $\nabla \mathbf{u}^{e}=\nabla \mathbf{u}^{e} \mathbf{P}$, with $\nabla \mathbf{u}:=\left(\nabla u_{1} \nabla u_{2} \nabla u_{3}\right)^{T} \in \mathbb{R}^{3 \times 3}$ for vector functions $\mathbf{u}$. The surface gradient and covariant derivatives on $\Gamma$ are then defined as $\nabla_{\Gamma} p=\mathbf{P} \nabla p^{e}$ and $\nabla_{\Gamma} \mathbf{u}:=\mathbf{P} \nabla \mathbf{u}^{e} \mathbf{P}$. Note that the definitions of surface gradient and covariant derivatives are independent of a particular smooth extension of $p$ and $\mathbf{u}$ off $\Gamma$. The reason why we consider normal extensions is because they are convenient for the error analysis. On $\Gamma$ we consider the surface rate-of-strain tensor [18] given by

$$
E_{s}(\mathbf{u}):=\frac{1}{2} \mathbf{P}\left(\nabla \mathbf{u}+\nabla \mathbf{u}^{T}\right) \mathbf{P}=\frac{1}{2}\left(\nabla_{\Gamma} \mathbf{u}+\nabla_{\Gamma} \mathbf{u}^{T}\right) .
$$

We also define the surface divergence operators for a vector $\mathbf{v}: \Gamma \rightarrow \mathbb{R}^{3}$ and a tensor A : $\Gamma \rightarrow \mathbb{R}^{3 \times 3}$ :

$$
\operatorname{div}_{\Gamma} \mathbf{v}:=\operatorname{tr}\left(\nabla_{\Gamma} \mathbf{v}\right), \quad \operatorname{div}_{\Gamma} \mathbf{A}:=\left(\operatorname{div}_{\Gamma}\left(\mathbf{e}_{1}^{T} \mathbf{A}\right), \operatorname{div}_{\Gamma}\left(\mathbf{e}_{2}^{T} \mathbf{A}\right), \operatorname{div}_{\Gamma}\left(\mathbf{e}_{3}^{T} \mathbf{A}\right)\right)^{T},
$$

with $\mathbf{e}_{i}$ the $i$ th basis vector in $\mathbb{R}^{3}$.

For a given force vector $\mathbf{f} \in L^{2}(\Gamma)^{3}$, with $\mathbf{f} \cdot \mathbf{n}=0$, and source term $g \in L^{2}(\Gamma)$, with $\int_{\Gamma} g d s=0$, we consider the following surface Stokes problem: Find a vector field $\mathbf{u}: \Gamma \rightarrow \mathbb{R}^{3}$, with $\mathbf{u} \cdot \mathbf{n}=0$, such that

$$
\begin{array}{rlrl}
-\mathbf{P} \operatorname{div}_{\Gamma}\left(E_{s}(\mathbf{u})\right)+\alpha \mathbf{u}+\nabla_{\Gamma} p & =\mathbf{f} & & \text { on } \Gamma, \\
\operatorname{div}_{\Gamma} \mathbf{u}=g & & \text { on } \Gamma,
\end{array}
$$

Here $\mathbf{u}$ is the tangential fluid velocity, $p$ the surface fluid pressure, and $\alpha \geq 0$ is a real parameter. The steady Stokes problem corresponds to $\alpha=0$, while $\alpha>0$ leads to a generalized Stokes problem, which results from an implicit time integration applied to a non-stationary Stokes equation ( $\alpha$ being proportional to the inverse of the time step). The non-zero source term $g$ in problem $\sqrt{2.2}-(2.3)$ is included to facilitate the treatment of evolving fluidic interfaces as a next research step. In that case, the 
inextensibility condition reads $\operatorname{div}_{\Gamma} \mathbf{u}_{T}=-u_{N} \kappa$, where $\kappa$ is the mean curvature and $u_{N}$ is the normal component of the velocity. Indeed, we use the velocity decompostion into tangential and normal components:

$$
\mathbf{u}=\mathbf{u}_{T}+u_{N} \mathbf{n}, \quad \mathbf{u}_{T} \cdot \mathbf{n}=0 .
$$

We remark that further in the text we use both $u_{N}$ and $\mathbf{n} \cdot \mathbf{u}$ to denote the normal compenent of the velocity $\mathbf{u}$. For the derivation of (Navier-)Stokes equations for evolving fluidic interfaces see, e.g., [24].

From problem $2.2-2.3$ one readily observes the following: the pressure field is defined up a hydrostatic mode; for $\alpha=0$ all tangentially rigid surface fluid motions, i.e. satisfying $E_{s}(\mathbf{u})=0$, are in the kernel of the differential operators at the lefthand side of eq. (2.2). Integration by parts implies the consistency condition for the right-hand side of eq. 2.2. :

$$
\int_{\Gamma} \mathbf{f} \cdot \mathbf{v} d s=0 \quad \text { for all smooth tangential vector fields } \mathbf{v} \text { s.t. } E_{s}(\mathbf{v})=\mathbf{0} .
$$

This condition is necessary for the well-posedness of problem $2.2-(2.3)$ when $\alpha=0$. In the literature a tangential vector field $\mathbf{v}$ defined on a surface and satisfying $E_{s}(\mathbf{v})=$ $\mathbf{0}$ is known as Killing vector field (cf., e.g., [39]). For a smooth two-dimensional Riemannian manifold, Killing vector fields form a Lie algebra of dimension 3 at most. The subspace of all the Killing vector fields on $\Gamma$ plays an important role in the analysis of the problem (2.2)-(2.3).

We introduce the following assumption:

Assumption 2.1. We assume that either no non-trivial Killing vector field exists on $\Gamma$ or $\alpha>0$.

REMARK 2.1. We briefly comment on assumption 2.1. If a non-trivial Killing vector field exists on $\Gamma$, then for the well-posedness of the surface Stokes problem (2.2)-(2.3) with $\alpha=0$ one has to restrict the velocity space to a suitable space that does not contain these Killing fields. If eq. 2.2 with $\alpha=0$ is understood as an equation for the equilibrium motion, i.e. the steady state, then the equilibrium solution is uniquely defined by an initial velocity $\mathbf{u}_{0}$. In fact, all tangentially rigid modes in $\mathbf{u}_{0}$ are conserved by the time-dependent surface Stokes equation. In order to find the unique equilibrium motion one has to consider a time-discretization for the time-dependent surface Stokes equation, that is equation 2.2 with $\alpha>0$. In order to avoid these (technical) difficulties for the case $\alpha=0$ and non-trivial Killing vector fields, we introduce assumption 2.1 .

REMARK 2.2. The operator $\mathbf{P} \operatorname{div}_{\Gamma} E_{s}(\cdot)$ in equation 2.2 models surface diffusion, which is a key component in modeling Newtonian surface fluids and fluidic membranes [40, 18, 4, 25, 24]. In the literature there are different formulations of the surface Navier-Stokes equations, some of which are formally obtained by substituting Cartesian differential operators by their geometric counterparts 43,8 . These formulations may involve different surface Laplace type operators, e.g., Hodge-de Rham Laplacian. We refer to 24 for a brief overview of different formulations of the surface Navier-Stokes equations.

For the weak formulation of problem (2.2)-(2.3), we introduce the space $\mathbf{V}:=$ $H^{1}(\Gamma)^{3}$ and norm

$$
\|\mathbf{u}\|_{1}^{2}:=\int_{\Gamma}\left(\|\mathbf{u}(s)\|^{2}+\left\|\nabla \mathbf{u}^{e}(s)\right\|^{2}\right) d s
$$


where $\|\cdot\|$ denotes the vector $\ell^{2}$-norm and the matrix Frobenius norm. We define the spaces

$$
\mathbf{V}_{T}:=\{\mathbf{u} \in \mathbf{V} \mid \mathbf{u} \cdot \mathbf{n}=0\}, \quad E:=\left\{\mathbf{u} \in \mathbf{V}_{T} \mid E_{s}(\mathbf{u})=\mathbf{0}\right\}
$$

Note that $E$ is a closed subspace of $\mathbf{V}_{T}$ and $\operatorname{dim}(E) \leq 3$. We define the Hilbert space $\mathbf{V}_{T}^{0}$ as an orthogonal complement of $E$ in $\mathbf{V}_{T}$ (hence $\mathbf{V}_{T}^{0} \sim \mathbf{V}_{T} / E$ ). For $\mathbf{u} \in \mathbf{V}$ we will use the orthogonal decomposition into tangential and normal parts as in (2.4). In what follows, we will need both general and tangential vector fields on $\Gamma$. Finally, we define $L_{0}^{2}(\Gamma):=\left\{p \in L^{2}(\Gamma) \mid \int_{\Gamma} p d x=0\right\}$.

Consider the bilinear forms (with $A: B=\operatorname{tr}\left(A B^{T}\right)$ for $A, B \in \mathbb{R}^{3 \times 3}$ )

$$
\begin{aligned}
a(\mathbf{u}, \mathbf{v}) & :=\int_{\Gamma}\left(E_{s}(\mathbf{u}): E_{s}(\mathbf{v})+\alpha \mathbf{u} \cdot \mathbf{v}\right) d s, \quad \mathbf{u}, \mathbf{v} \in \mathbf{V}, \\
b_{T}(\mathbf{u}, p) & :=-\int_{\Gamma} p \operatorname{div}_{\Gamma} \mathbf{u}_{T} d s, \quad \mathbf{u} \in \mathbf{V}, p \in L^{2}(\Gamma) .
\end{aligned}
$$

We emphasize that in the definition of $b_{T}(\mathbf{u}, p)$ only the tangential component of $\mathbf{u}$ is used, i.e., $b_{T}(\mathbf{u}, p)=b_{T}\left(\mathbf{u}_{T}, p\right)$ for all $\mathbf{u} \in \mathbf{V}, p \in L^{2}(\Gamma)$. This property motivates the notation $b_{T}(\cdot, \cdot)$ instead of $b(\cdot, \cdot)$.

The weak (variational) formulation of the surface Stokes problem (2.2)-(2.3) reads: Determine $\left(\mathbf{u}_{T}, p\right) \in \mathbf{V}_{T} \times L_{0}^{2}(\Gamma)$ such that

$$
\begin{aligned}
a\left(\mathbf{u}_{T}, \mathbf{v}_{T}\right)+b_{T}\left(\mathbf{v}_{T}, p\right) & =\left(\mathbf{f}, \mathbf{v}_{T}\right)_{L^{2}} \quad \text { for all } \mathbf{v}_{T} \in \mathbf{V}_{T} \\
b_{T}(\mathbf{u}, q) & =(g, q)_{L^{2}} \quad \text { for all } q \in L^{2}(\Gamma) .
\end{aligned}
$$

Here, $(\cdot, \cdot)_{L^{2}}$ denotes the $L^{2}$ scalar product on $\Gamma$. The following surface Korn inequality and inf-sup property were derived in 24.

Lemma 2.1. Assume $\Gamma$ is $C^{2}$ smooth and compact. There exist $c_{K}>0$ and $c_{0}>0$ such that

$$
\left\|E_{s}\left(\mathbf{v}_{T}\right)\right\|_{L^{2}} \geq c_{K}\left\|\mathbf{v}_{T}\right\|_{1} \text { for all } \mathbf{v}_{T} \in \mathbf{V}_{T}^{0}
$$

and

$$
\sup _{\mathbf{v}_{T} \in \mathbf{V}_{T}^{0}} \frac{b_{T}\left(\mathbf{v}_{T}, p\right)}{\left\|\mathbf{v}_{T}\right\|_{1}} \geq c_{0}\|p\|_{L^{2}} \quad \text { for all } p \in L_{0}^{2}(\Gamma)
$$

Since $E$ is finite dimensional (and so all norms on $E$ are equivalent), inequality 2.12 implies

$$
\left\|\mathbf{v}_{T}\right\|_{L^{2}}+\left\|E_{s}\left(\mathbf{v}_{T}\right)\right\|_{L^{2}} \geq c_{K}\left\|\mathbf{v}_{T}\right\|_{1} \quad \text { for all } \mathbf{v}_{T} \in \mathbf{V}_{T} .
$$

Using 2.12 and Assumption 2.1 for $\alpha=0$ and 2.14 for $\alpha>0$ we obtain the norm equivalence

$$
a\left(\mathbf{v}_{T}, \mathbf{v}_{T}\right) \simeq\left\|\mathbf{v}_{T}\right\|_{1}^{2} \text { for all } \mathbf{v}_{T} \in \mathbf{V}_{T} .
$$

Inf-sup stability of $b_{T}(\cdot, \cdot)$ on $\mathbf{V}_{T} \times L_{0}^{2}(\Gamma)$ follows from 2.13$)$. Both bilinear forms $a(\cdot, \cdot)$ and $b_{T}(\cdot, \cdot)$ are continuous. Therefore problem (2.10)-(2.11) is well posed, and its unique solution is further denoted by $\left\{\mathbf{u}_{T}^{*}, p^{*}\right\}$. 
3. Penalty formulation. The weak formulation 2.10 -2.11 is not very suited for a Galerkin finite element discretization, since it requires finite element functions that are tangential to $\Gamma$. Such functions are not easy to construct. Thus, following 21, 22, 24, 38, we consider a variational formulation in a larger space $\mathbf{V}_{*} \supset \mathbf{V}_{T}$, introduced below, augmented by a penalty term to enforce the tangential constraint weakly. This variational method will be the basis for the finite element method introduced in section 4 .

In order to write the alternative variational formulation, we introduce the following Hilbert space and corresponding norm:

$$
\mathbf{V}_{*}:=\left\{\mathbf{u} \in L^{2}(\Gamma)^{3}: \mathbf{u}_{T} \in \mathbf{V}_{T}, u_{N} \in L^{2}(\Gamma)\right\}, \quad \text { with }\|\mathbf{u}\|_{V_{*}}^{2}:=\left\|\mathbf{u}_{T}\right\|_{1}^{2}+\tau\left\|u_{N}\right\|_{L^{2}}^{2},
$$

where $\tau$ is a positive real parameter. We also report the following useful relation:

$$
E_{s}(\mathbf{u})=E_{s}\left(\mathbf{u}_{T}\right)+u_{N} \mathbf{H},
$$

where $\mathbf{H}:=\nabla_{\Gamma} \mathbf{n}$ is the shape operator (second fundamental form) on $\Gamma$. Let us define the bilinear form

$$
a_{\tau}(\mathbf{u}, \mathbf{v}):=\int_{\Gamma}\left(E_{s}(\mathbf{u}): E_{s}(\mathbf{v})+\alpha \mathbf{u}_{T} \cdot \mathbf{v}_{T}\right) d s+\tau \int_{\Gamma} u_{N} v_{N} d s
$$

for $\mathbf{u}, \mathbf{v} \in \mathbf{V}_{*}$. Using (3.1) we can rewrite it as

$$
\begin{aligned}
a_{\tau}(\mathbf{u}, \mathbf{v})= & a\left(\mathbf{u}_{T}, \mathbf{v}_{T}\right)+\int_{\Gamma} E_{s}\left(\mathbf{u}_{T}\right): v_{N} \mathbf{H} d s+\int_{\Gamma} E_{s}\left(\mathbf{v}_{T}\right): u_{N} \mathbf{H} d s \\
& +\left(\|\mathbf{H}\|^{2} u_{N}, v_{N}\right)_{L^{2}}+\tau\left(u_{N}, v_{N}\right)_{L^{2}},
\end{aligned}
$$

which is well-defined on $\mathbf{V}_{*} \times \mathbf{V}_{*}$. In $3.2, \tau$ is an augmentation (penalty) parameter. Using

$$
2 \int_{\Gamma} E_{s}\left(\mathbf{u}_{T}\right): u_{N} \mathbf{H} d s \geq-\frac{1}{2}\left\|E_{s}\left(\mathbf{u}_{T}\right)\right\|_{L^{2}}^{2}-2\|\mathbf{H}\| u_{N} \|_{L^{2}}^{2}
$$

and 2.15 we conclude that if $\tau \geq \max \left\{1,2\|\mathbf{H}\|_{L^{\infty}(\Gamma)}^{2}\right\}$, there are constants $c_{0}>0$, $c_{1}$, independent of $\tau$ such that

$$
c_{0}\|\mathbf{u}\|_{V_{*}}^{2} \leq a_{\tau}(\mathbf{u}, \mathbf{u}) \leq c_{1}\|\mathbf{u}\|_{V_{*}}^{2} \text { for all } \mathbf{u} \in V_{*} .
$$
holds.

Assumption 3.1. In the remainder we assume that $\tau \geq \max \left\{1,2\|\mathbf{H}\|_{L^{\infty}(\Gamma)}^{2}\right\}$

The alternative variational formulation reads: Find $(\hat{\mathbf{u}}, \hat{p}) \in \mathbf{V}_{*} \times L_{0}^{2}(\Gamma)$ such that

$$
\begin{aligned}
& a_{\tau}(\hat{\mathbf{u}}, \mathbf{v})+b_{T}(\mathbf{v}, \hat{p})=(\mathbf{f}, \mathbf{v})_{L^{2}} \quad \text { for all } \mathbf{v} \in \mathbf{V}_{*} \\
& b_{T}(\hat{\mathbf{u}}, q)=(g, q)_{L^{2}} \quad \text { for all } q \in L^{2}(\Gamma) .
\end{aligned}
$$

Well-posedness of the augmented surface Stokes problem $(3.5)-(3.6)$ and an estimate on the difference between its solution and the solution to (2.10)-(2.11) are given in the following theorem, which extends a result in [24].

TheOrem 3.1. Problem (3.5)-(3.6) is well posed. For the unique solution $(\hat{\mathbf{u}}, \hat{p}) \in$ $\mathbf{V}_{*} \times L_{0}^{2}(\Gamma)$ of this problem and the unique solution $\left(\mathbf{u}_{T}^{*}, p^{*}\right) \in \mathbf{V}_{T} \times L_{0}^{2}(\Gamma)$ of $(2.10$ 2.11) the following estimate holds

$$
\left\|\hat{\mathbf{u}}_{T}-\mathbf{u}_{T}^{*}\right\|_{1}+\left\|\hat{u}_{N}\right\|_{L^{2}}+\left\|\hat{p}-p^{*}\right\|_{L^{2}} \leq C \tau^{-1}\left(\|\mathbf{f}\|_{L^{2}}+\|g\|_{L^{2}}\right),
$$


where $C$ depends only on $\Gamma$.

Proof. The bilinear form $a_{\tau}(\cdot, \cdot)$ is continuous and elliptic on $\mathbf{V}_{*}$, as shown in (3.4). The uniform in $\tau$ inf-sup property and continuity for $b_{T}(\cdot, \cdot)$ on $\mathbf{V}_{*} \times L_{0}^{2}(\Gamma)$ immediately follow from 2.13), the embedding $\mathbf{V}_{T}^{0} \subset \mathbf{V}_{*}$ and the property $b_{T}(\mathbf{v}, q)=b_{T}\left(\mathbf{v}_{T}, q\right)$. Therefore, problem (3.5)-3.6) is well posed and the following a priori estimate holds

$$
\|\hat{\mathbf{u}}\|_{V_{*}}+\|\hat{p}\|_{L^{2}} \leq c\left(\|\mathbf{f}\|_{\mathbf{V}_{*}^{\prime}}+\|g\|_{L^{2}}\right) \leq c\left(\|\mathbf{f}\|_{L^{2}}+\|g\|_{L^{2}}\right),
$$

with some $c$ independent of $\tau$ and $\mathbf{f}, g$.

We test equation (3.5) with $\mathbf{v}=\hat{u}_{N} \mathbf{n}$. Thanks to (3.1) and $\mathbf{f} \cdot \mathbf{n}=0$, we obtain the identity

$$
\int_{\Gamma} E_{s}(\hat{\mathbf{u}}): \hat{u}_{N} \mathbf{H} d s+\tau\left\|\hat{u}_{N}\right\|_{L^{2}}^{2}=0 .
$$

Then, the Cauchy inequality and inequality (3.8) lead to

$$
\begin{aligned}
\tau\left\|\hat{u}_{N}\right\|_{L^{2}}^{2} & =-\int_{\Gamma} E_{s}(\hat{\mathbf{u}}): \hat{u}_{N} \mathbf{H} d s \leq C\left\|\hat{u}_{N}\right\|_{L^{2}}\left\|E_{s}(\hat{\mathbf{u}})\right\|_{L^{2}} \leq C\left\|\hat{u}_{N}\right\|_{L^{2}}\|\hat{\mathbf{u}}\|_{V_{*}} \\
& \leq C\left\|\hat{u}_{N}\right\|_{L^{2}}\left(\|\mathbf{f}\|_{L^{2}}+\|g\|_{L^{2}}\right) .
\end{aligned}
$$

Hence, we proved the desired estimate for $\left\|\hat{u}_{N}\right\|_{L^{2}}$. We now consider the term $\| \hat{\mathbf{u}}_{T}-$ $\mathbf{u}_{T}^{*} \|_{1}$. We take $\mathbf{v}_{T}:=\hat{\mathbf{u}}_{T}-\mathbf{u}_{T}^{*}$ in equations (2.10)-(2.11) and (3.5)-(3.6). From the divergence equations in (2.11) and (3.6) we obtain $b_{T}\left(\mathbf{v}_{T}, q\right)=0$ for all $q \in L^{2}(\Gamma)$. Taking $\mathbf{v}=\mathbf{v}_{T}$ in (2.10) and (3.5) and using $b_{T}\left(\mathbf{v}_{T}, q\right)=0$ for all $q \in L^{2}(\Gamma)$ we get

$$
a\left(\mathbf{u}_{T}^{*}, \mathbf{v}_{T}\right)-a_{\tau}\left(\hat{\mathbf{u}}, \mathbf{v}_{T}\right)=0
$$

and using $(3.3)$ we obtain

$$
a\left(\mathbf{v}_{T}, \mathbf{v}_{T}\right)=-\int_{\Gamma} E_{s}\left(\mathbf{v}_{T}\right): \hat{u}_{N} \mathbf{H} d s
$$

From this and 2.15) we conclude

$$
\left\|\mathbf{u}_{T}^{*}-\hat{\mathbf{u}}_{T}\right\|_{1}^{2} \leq c a\left(\mathbf{v}_{T}, \mathbf{v}_{T}\right) \leq c\left\|\mathbf{u}_{T}^{*}-\hat{\mathbf{u}}_{T}\right\|_{1}\left\|\hat{u}_{N}\right\|_{L^{2}} .
$$

Hence, $\left\|\mathbf{u}_{T}^{*}-\hat{\mathbf{u}}_{T}\right\|_{1} \leq c\left\|\hat{u}_{N}\right\|_{L^{2}} \leq c \tau^{-1}\left(\|\mathbf{f}\|_{L^{2}}+\|g\|_{L^{2}}\right)$ holds, which is the desired estimate for $\left\|\hat{\mathbf{u}}_{T}-\mathbf{u}_{T}^{*}\right\|_{1}$. Finally, the estimate for $\left\|\hat{p}-p^{*}\right\|_{L^{2}}$ follows from inequalities (2.13), (3.9), and (3.10):

$$
\begin{aligned}
c\left\|p^{*}-\hat{p}\right\|_{L^{2}} & \leq \sup _{\mathbf{v}_{T} \in \mathbf{V}_{T}^{0}} \frac{b_{T}\left(\mathbf{v}_{T}, p^{*}-\hat{p}\right)}{\left\|\mathbf{v}_{T}\right\|_{1}}=\sup _{\mathbf{v}_{T} \in \mathbf{V}_{T}^{0}} \frac{a\left(\mathbf{u}_{T}^{*}, \mathbf{v}_{T}\right)-a_{\tau}\left(\hat{\mathbf{u}}, \mathbf{v}_{T}\right)}{\left\|\mathbf{v}_{T}\right\|_{1}} \\
& =\sup _{\mathbf{v}_{T} \in \mathbf{V}_{T}^{0}} \frac{a\left(\mathbf{u}_{T}^{*}-\hat{\mathbf{u}}_{T}, \mathbf{v}_{T}\right)-\int_{\Gamma} E_{s}\left(\mathbf{v}_{T}\right): \hat{u}_{N} \mathbf{H} d s}{\left\|\mathbf{v}_{T}\right\|_{1}} \\
& \leq C\left(\left\|\hat{\mathbf{u}}_{T}-\mathbf{u}_{T}^{*}\right\|_{1}+\left\|\hat{u}_{N}\right\|_{L^{2}}\right) \leq C \tau^{-1}\left(\|\mathbf{f}\|_{L^{2}}+\|g\|_{L^{2}}\right) .
\end{aligned}
$$

This concludes the proof.

If one is interested in a finite element method of order $m$ for the surface Stokes problem 2.2 - 2.3), then Theorem 3.1 suggests to use weak formulation (3.5)-3.6 
with penalty parameter $\tau=O\left(h^{-m}\right)$. For the particular choice of $P_{1}-P_{1}$ elements used in this paper, this motivates

$$
\tau=c_{\tau} h^{-2}
$$

with some $c_{\tau}$ depending only on $\Gamma$.

We conclude by stressing again that the weak formulation (3.5)-(3.6) gives a numerical advantage over formulation (2.10)-2.11) by not forcing the use of tangential finite element vector fields.

4. Trace Finite Element Method. For the discretization of the variational problem (3.5)-(3.6) we extend the trace finite element approach (TraceFEM) introduced in [30] for elliptic equations on surfaces. In this section, we present and analyze the method.

Let $\Omega \subset \mathbb{R}^{3}$ be a fixed polygonal domain that strictly contains $\Gamma$. We consider a family of shape regular tetrahedral triangulations $\left\{\mathcal{T}_{h}\right\}_{h>0}$ of $\Omega$. The subset of tetrahedra that have a nonzero intersection with $\Gamma$ is collected in the set denoted by $\mathcal{T}_{h}^{\Gamma}$. For the analysis of the method, we assume $\left\{\mathcal{T}_{h}^{\Gamma}\right\}_{h>0}$ to be quasi-uniform. However, in practice adaptive mesh refinement is possible, as discussed, for example, in [10, 9]. The domain formed by all tetrahedra in $\mathcal{T}_{h}^{\Gamma}$ is denoted by $\Omega_{h}^{\Gamma}:=\operatorname{int}\left(\overline{\cup_{T \in \mathcal{T}_{h}^{\Gamma}} T}\right)$. On $\mathcal{T}_{h}^{\Gamma}$ we use a standard finite element space of continuous functions that are piecewiseaffine functions. In this paper we focus on $P_{1}$ elements, i.e. polynomials of degree 1 . This so-called bulk finite element space is denoted by $V_{h}$.

Since the $P_{1}-P_{1}$ pair for velocity and pressure is not inf-sup stable, a stabilization term is added to the finite element (FE) formulation (see below). For this extra term, we need an extension of the normal vector field $\mathbf{n}$ from $\Gamma$ to $\Omega_{h}^{\Gamma}$, denoted with $\mathbf{n}^{e}$. We choose $\mathbf{n}^{e}=\nabla d$, where $d$ is the signed distance function to $\Gamma$. In practice, $d$ is often not available and thus we use approximations, as discussed in Remark 4.1. Another implementation aspect of TraceFEM that requires attention is the computation of integrals over the surface $\Gamma$ with sufficiently high accuracy. In practice, $\Gamma$ can be defined implicitly as the zero level of a level set function and a parametrization of $\Gamma$ may not be available. An easy way to compute approximation $\Gamma_{h} \approx \Gamma$ and the corresponding geometric errors will also be discussed in Remark 4.1. Below we use the exact extended normal $\mathbf{n}=\mathbf{n}^{e}$ and we assume exact integration over $\Gamma$.

Consider the spaces

$$
\mathbf{U}:=\left\{\mathbf{v} \in H^{1}\left(\Omega_{h}^{\Gamma}\right)^{3}|\mathbf{v}|_{\Gamma} \in \mathbf{V}_{*}\right\}, \quad Q:=H^{1}\left(\Omega_{h}^{\Gamma}\right) .
$$

Our velocity and pressure finite element spaces are $P_{1}$ continuous FE spaces on $\Omega_{h}^{\Gamma}$.

$$
\mathbf{U}_{h}:=\left(V_{h}\right)^{3} \subset \mathbf{U}, \quad Q_{h}:=V_{h} \cap L_{2}^{0}(\Gamma) \subset Q .
$$

We introduce the following finite element bilinear forms:

$$
\begin{aligned}
A_{h}(\mathbf{u}, \mathbf{v}) & :=a_{\tau}(\mathbf{u}, \mathbf{v})+\rho_{u} \int_{\Omega_{h}^{\Gamma}}(\nabla \mathbf{u n}) \cdot(\nabla \mathbf{v n}) d x, \quad \mathbf{u}, \mathbf{v} \in \mathbf{U}, \\
s_{h}(p, q) & :=\rho_{p} \int_{\Omega_{h}^{\Gamma}} \nabla p \cdot \nabla q d x, \quad p, q \in Q .
\end{aligned}
$$

The volumetric term in the definition of $A_{h}$ is the so called volume normal derivative stabilization first introduced in [7, 16] in the context of TraceFEM for the scalar 
Laplace-Beltrami problem on a surface. The term vanishes for the strong solution $\mathbf{u}$ of equations (2.2)-(2.3), since one can always assume a normal extension of $\mathbf{u}$ off the surface. The purpose of this additional term is to stabilize the resulting algebraic system against possible instabilities produced by the small cuts of the background triangulation by the surface. Indeed, if one uses a natural nodal basis in $V_{h}$, then small cuts of background tetrahedra may lead to (arbitrarily) small diagonal entries in the resulting matrices. The stabilization term in 4.1) eliminates this problem because it allows to get control over the $L^{2}\left(\Omega_{h}^{\Gamma}\right)$-norm of $\mathbf{v}_{h} \in \mathbf{U}_{h}$ by the problem induced norm $A_{h}\left(\mathbf{v}_{h}, \mathbf{v}_{h}\right)^{\frac{1}{2}}$ for a suitable choice of $\rho_{u}$. We note that other efficient stabilization techniques exist; see [7] and the review in [31.

The role of $s_{h}$ defined in 4.2 is twofold. First, it stabilizes the nodal basis in the pressure space $Q_{h}$ with respect to small element cuts, in the same way as the volumetric term in (4.1) does this for velocity. Then, it stabilizes the velocity-pressure pair against the violation of the inf-sup condition (the discrete counterpart of (2.13). For the latter, the $s_{h}$ stabilization resembles the well-known Brezzi-Pitkäranta stabilization [6] for the planar Stokes $P_{1}-P_{1}$ finite elements. Both roles are clearly seen from the decomposition:

$$
s_{h}(p, q)=\underbrace{\rho_{p} \int_{\Omega_{h}^{\Gamma}} \frac{\partial p}{\partial \mathbf{n}} \frac{\partial q}{\partial \mathbf{n}} d x}_{\text {normal stabilization }}+\underbrace{\rho_{p} \int_{\Omega_{h}^{\Gamma}} \nabla_{\Gamma} p \nabla_{\Gamma} q d x}_{\text {Brezzi-Pitkäranta stabilization }} .
$$

The analysis of the scalar Laplace-Beltrami surface equation [7, 16, and vector surface Laplacians [17] suggest that optimal convergence and algebraic stability should be expected for a wide range of the normal stabilization parameter, $h \lesssim \rho_{u} \lesssim h^{-1}$. In this paper, we introduce the minimal suitable stabilization and set $\rho_{p} \simeq \rho_{u} \simeq h$. Here and further in the paper we write $x \lesssim y$ to state that the inequality $x \leq c y$ holds for quantities $x, y$ with a constant $c$, which is independent of the mesh parameter $h$ and the position of $\Gamma$ over the background mesh. Similarly for $x \gtrsim y$, and $x \simeq y$ will mean that both $x \lesssim y$ and $x \gtrsim y$ hold. Note that a $\rho_{p} \simeq h$ scaling is consistent with the well-known $O\left(h^{2}\right)$ choice of the Brezzi-Pitkäranta stabilization parameter in the usual (planar or volumetric) case. The additional $O(h)$ scaling comes from the fact that the second term in 4.3 is computed over the narrow volumetric domain rather than over the surface.

The trace finite element method (TraceFEM) we use reads as follows: Find $\left(\mathbf{u}_{h}, p_{h}\right) \in \mathbf{U}_{h} \times Q_{h}$ such that

$$
\begin{aligned}
A_{h}\left(\mathbf{u}_{h}, \mathbf{v}_{h}\right)+b_{T}\left(\mathbf{v}_{h}, p_{h}\right) & =\left(\mathbf{f}, \mathbf{v}_{h}\right)_{L^{2}} & & \text { for all } \mathbf{v}_{h} \in \mathbf{U}_{h} \\
b_{T}\left(\mathbf{u}_{h}, q_{h}\right)-s_{h}\left(p_{h}, q_{h}\right) & =\left(g, q_{h}\right)_{L^{2}} & & \text { for all } q_{h} \in Q_{h},
\end{aligned}
$$

with the following setting for the parameters:

$$
\tau=c_{\tau} h^{-2}, \quad \rho_{p}=c_{p} h, \quad \rho_{u}=c_{u} h .
$$

Here $h$ is the characteristic mesh size of the background tetrahedral mesh, while $c_{\tau}$, $c_{p}, c_{u}$ are some $O(1)$ tunable constants. The optimal value of those constants may depend on problem data such as $\Gamma$, but is independent of $h$ and of how $\Gamma$ cuts through the background mesh. The decomposition (4.3) suggests that one can split $s_{h}(\cdot, \cdot)$ into two parts and use different scalings for the normal and tangential terms. For simplicity of the method, we avoid this option.

REMARK 4.1. We discuss some implementation aspects of the trace finite element discretization (4.4). In the bilinear form $A_{h}\left(\mathbf{u}_{h}, \mathbf{v}_{h}\right)$ only full gradients (no 
tangential ones) of the arguments are needed. These can be computed as in standard finite element methods. It is important for the implementation that in $A_{h}(\cdot, \cdot)$ we do not need derivatives of projected velocities, e.g. of $\left(\mathbf{u}_{h}\right)_{T}$. In the bilinear form $b_{T}\left(\mathbf{v}_{h}, p_{h}\right)=-\int_{\Gamma} p_{h} \operatorname{div}_{\Gamma}\left(\mathbf{v}_{h}\right)_{T} d s$, however, derivatives of the tangential velocity $\left(\mathbf{v}_{h}\right)_{T}=\mathbf{P} \mathbf{v}_{h}$, with $\mathbf{P}:=\mathbf{I}-\mathbf{n n} \mathbf{n}^{T}$, appear. This requires a differentation of $\mathbf{P}$ and thus a sufficiently accurate curvature approximation. In a setting of $H^{1}$-conforming pressure finite element spaces, as used in this paper, it is convenient to rewrite the bilinear form as $b_{T}\left(\mathbf{v}_{h}, p_{h}\right)=\int_{\Gamma} \nabla_{\Gamma} p_{h} \cdot \mathbf{v}_{h} d s=\int_{\Gamma}\left(\mathbf{P} \nabla p_{h}\right) \cdot \mathbf{v}_{h} d s$. Implementation then only requires an approximation of $\mathbf{n}_{h} \approx \mathbf{n}$ and not of derivatives of $\mathbf{n}$.

As noted above, in the implementation of this method one typically replaces $\Gamma$ by an approximation $\Gamma_{h} \approx \Gamma$ such that integrals over $\Gamma_{h}$ can be efficiently computed. Furthermore, the exact normal $\mathbf{n}$ is approximated by $\mathbf{n}_{h} \approx \mathbf{n}$. In the literature on finite element methods for surface PDEs, this is standard practice. We will use a piecewise planar surface approximation $\Gamma_{h}$ with $\operatorname{dist}\left(\Gamma, \Gamma_{h}\right) \lesssim h^{2}$. If one is interested in surface FEM with higher order surface approximation, we refer to the recent paper [16. We assume a level set representation of $\Gamma$ :

$$
\Gamma=\left\{\mathbf{x} \in \mathbb{R}^{3}: \phi(\mathbf{x})=0\right\},
$$

with some smooth function $\phi$ such that $|\nabla \phi| \geq c_{0}>0$ in a neighborhood of $\Gamma$. For the numerical experiments in section 7 we use a piecewise planar surface approximation:

$$
\Gamma_{h}=\left\{\mathbf{x} \in \mathbb{R}^{3}: I_{h}(\phi(\mathbf{x}))=0\right\},
$$

where $I_{h}(\phi(\mathbf{x})) \in V_{h}$ is the nodal interpolant of $\phi$. As for the construction of suitable normal approximations $\mathbf{n}_{h} \approx \mathbf{n}$, several techniques are available in the literature. One possibility is to use $\mathbf{n}_{h}(\mathbf{x})=\frac{\nabla \phi_{h}(\mathbf{x})}{\left\|\nabla \phi_{h}(\mathbf{x})\right\|_{2}}$, where $\phi_{h}$ is a finite element approximation of a level set function $\phi$ which characterizes $\Gamma$. This is technique we use in section 7 , where $\phi_{h}$ is defined as a $P_{2}$ nodal interpolant of $\phi$. Analyzing the effect of such geometric errors is beyond the scope of this paper. Our focus is on analyzing the TraceFEM 4.4 .

REMARK 4.2. An alternative numerical approach to enforce the tangentiality constraint on the flow field $\mathbf{u}$ is to introduce a Lagrange multiplier in (4.4) instead of using a penalty approach. This adds extra Lagrange multiplier unknowns to the algebraic system, but removes the augmentation parameter $\tau$. For a surface vectorLaplace problem the TraceFEM with such an enforcement of $\mathbf{u} \cdot \mathbf{n}=0$ was studied in [17. For $P_{1}$ velocity elements one can use a $P_{1}$ Lagrange multiplier space for a numerically stable and optimally accurate TraceFEM. A systematic comparison of the penalty approach presented in this paper and such a Lagrange multiplier technique for the surface Stokes problem is a topic for future research.

5. Error analysis of TraceFEM. In this section we present stability and error analysis of the finite element method (4.4). After some preliminaries we derive a discrete inf-sup result and discuss the consistency between the FE formulation and the original problem. We then prove an $O(h)$ error estimate in the natural energy norm and an $O\left(h^{2}\right)$ error estimate in the surface $L^{2}$-norm for velocity.

5.1. Preliminaries. In this section we collect a few results that we need in the error analysis. The parameters in the bilinear forms are set as in 4.5). We introduce 
the norms:

$$
\begin{aligned}
\|\mathbf{v}\|_{U}^{2} & :=A_{h}(\mathbf{v}, \mathbf{v}), \quad \mathbf{v} \in \mathbf{U}, \\
\|q\|_{Q}^{2} & :=\|q\|_{L^{2}}^{2}+h\|\nabla q\|_{L^{2}\left(\Omega_{h}^{\Gamma}\right)}^{2}, \quad q \in Q .
\end{aligned}
$$

In these norms we easily obtain continuity estimates. The Cauchy-Schwarz inequality and the definition of the norms immediately yield the following estimates:

$$
\begin{aligned}
& A_{h}(\mathbf{u}, \mathbf{v}) \leq\|\mathbf{u}\|_{U}\|\mathbf{v}\|_{U} \quad \text { for all } \mathbf{u}, \mathbf{v} \in \mathbf{U}, \\
& b_{T}(\mathbf{u}, q) \lesssim\|\mathbf{u}\|_{U}\|q\|_{Q} \quad \text { for all } \mathbf{u} \in \mathbf{U}, q \in Q \text {. } \\
& s_{h}(p, q) \lesssim\|p\|_{Q}\|q\|_{Q} \quad \text { for all } p, q \in Q \text {. }
\end{aligned}
$$

On the finite element spaces $\mathbf{U}_{h}, Q_{h}$ the norms $\|\cdot\|_{U}$ and $\|\cdot\|_{Q}$ are uniformly equivalent to certain (scaled) $L^{2}$ and $H^{1}$ norms. The uniformity holds with respect to $h$ and the position of $\Gamma$ in the background mesh. We recall a result known from the literature for scalar finite element function $v_{h} \in V_{h}$ :

$$
h\left\|v_{h}\right\|_{L^{2}}^{2}+h^{2}\left\|\mathbf{n} \cdot \nabla v_{h}\right\|_{L^{2}\left(\Omega_{h}^{\Gamma}\right)}^{2} \simeq\left\|v_{h}\right\|_{L^{2}\left(\Omega_{h}^{\Gamma}\right)}^{2} \quad \text { for all } v_{h} \in V_{h} .
$$

A proof of the $\gtrsim$ estimate in 5.6 is given in [16, while the $\lesssim$ estimate follows from the inequality, cf. [20]:

$$
h\|v\|_{L^{2}(\Gamma \cap K)}^{2} \lesssim\|v\|_{L^{2}(K)}^{2}+h^{2}\|v\|_{H^{1}(K)}^{2} \quad \text { for all } v \in H^{1}(K), K \in \mathcal{T}_{h}^{\Gamma},
$$

and a standard finite element inverse inequality $\left\|v_{h}\right\|_{H^{1}\left(\Omega_{h}^{\Gamma}\right)} \lesssim h^{-1}\left\|v_{h}\right\|_{L^{2}\left(\Omega_{h}^{\Gamma}\right)}$ for all $v_{h} \in V_{h}$. Using (5.6), (3.4) and 4.5) we get for vector functions $\mathbf{v}=\left(v_{1}, v_{2}, v_{3}\right) \in \mathbf{U}_{h}$ :

$$
\begin{aligned}
A_{h}(\mathbf{v}, \mathbf{v}) & \simeq\|\mathbf{v}\|_{V_{*}}^{2}+h \sum_{i=1}^{3}\left\|\mathbf{n} \cdot \nabla v_{i}\right\|_{L^{2}\left(\Omega_{h}^{\Gamma}\right)}^{2} \\
& \simeq\left\|\mathbf{v}_{T}\right\|_{1}^{2}+h^{-2}\left\|v_{N}\right\|_{L^{2}}^{2}+\sum_{i=1}^{3}\left(\left\|v_{i}\right\|_{L^{2}}^{2}+h\left\|\mathbf{n} \cdot \nabla v_{i}\right\|_{L^{2}\left(\Omega_{h}^{\Gamma}\right)}^{2}\right) \\
& \simeq\left\|\mathbf{v}_{T}\right\|_{1}^{2}+h^{-2}\left\|v_{N}\right\|_{L^{2}}^{2}+h^{-1}\|\mathbf{v}\|_{L^{2}\left(\Omega_{h}^{\Gamma}\right)}^{2},
\end{aligned}
$$

i.e.:

$$
\|\mathbf{v}\|_{U}^{2} \simeq\left\|\mathbf{v}_{T}\right\|_{1}^{2}+h^{-2}\left\|v_{N}\right\|_{L^{2}}^{2}+h^{-1}\|\mathbf{v}\|_{L^{2}\left(\Omega_{h}^{\Gamma}\right)}^{2} \quad \text { for all } \mathbf{v} \in \mathbf{U}_{h} .
$$

From 5.6 and finite element inverse inequalities we also obtain:

$$
\|q\|_{Q} \simeq h^{-\frac{1}{2}}\|q\|_{L^{2}\left(\Omega_{h}^{\Gamma}\right)} \quad \text { for all } q \in Q_{h} .
$$

5.2. Discrete inf-sup property. Based on the inf-sup property (2.13) for the continuous problem and a perturbation argument we derive a discrete inf-sup result. In the analysis, for a scalar function $v \in L^{2}(\Gamma)$ we use a constant extension along normals denoted by $v^{e}$, which is defined in a fixed sufficiently small neighborhood of $\Gamma$ that contains (for $h$ sufficiently small) the local triangulation $\Omega_{h}^{\Gamma}$, cf. [11. For $v^{e}$ the following estimates hold [35]:

$$
\begin{aligned}
& h^{\frac{1}{2}}\left\|\nabla_{\Gamma} v\right\|_{L^{2}} \simeq\left\|\nabla v^{e}\right\|_{L^{2}\left(\Omega_{h}^{\Gamma}\right)}, \quad \text { for all } v \in H^{1}(\Gamma), \\
& h^{\frac{1}{2}}\|v\|_{L^{2}} \simeq\left\|v^{e}\right\|_{L^{2}\left(\Omega_{h}^{\Gamma}\right)}, \quad \text { for all } v \in L^{2}(\Gamma), \\
& \left\|v^{e}\right\|_{H^{2}\left(\Omega_{h}^{\Gamma}\right)} \lesssim h^{\frac{1}{2}}\|v\|_{H^{2}(\Gamma)}, \quad \text { for all } v \in H^{2}(\Gamma) \text {. }
\end{aligned}
$$


The componentwise constant extension along normals of a vector function $\mathbf{v}$ is denoted by $\mathbf{v}^{e}$. Applying the first estimate in 5.10 componentwise we also get for all $\mathbf{v} \in$ $H^{1}(\Gamma)^{3}$ :

$$
h^{\frac{1}{2}}\|\nabla \mathbf{v P}\|_{L^{2}} \simeq\left\|\nabla \mathbf{v}^{e}\right\|_{L^{2}\left(\Omega_{h}^{\Gamma}\right)}, \text { and thus }\left\|\nabla \mathbf{v}^{e}\right\|_{L^{2}\left(\Omega_{h}^{\Gamma}\right)} \lesssim h^{\frac{1}{2}}\|\mathbf{v}\|_{1} .
$$

Lemma 5.1. If the constant $c_{p}$ in 4.5 is taken sufficiently large (independent of $h$ and of how $\Gamma$ intersects the background mesh) then the following holds:

$$
\sup _{\mathbf{v}_{h} \in \mathbf{U}_{h}} \frac{b_{T}\left(\mathbf{v}_{h}, q_{h}\right)}{\left\|\mathbf{v}_{h}\right\|_{U}}+s_{h}\left(q_{h}, q_{h}\right)^{\frac{1}{2}} \gtrsim\left\|q_{h}\right\|_{Q} \quad \text { for all } q_{h} \in Q_{h} .
$$

Proof. Take $q_{h} \in Q_{h}$. Thanks to the inf-sup property (2.13), there exists $\mathbf{v} \in \mathbf{V}_{T}$ such that

$$
b_{T}\left(\mathbf{v}, q_{h}\right)=\left\|q_{h}\right\|_{L^{2}}^{2}, \quad c_{0}\|\mathbf{v}\|_{1} \leq\left\|q_{h}\right\|_{L^{2}} .
$$

Let $\mathbf{v}^{e}$ be the normal extension of $\mathbf{v}$ and take $\mathbf{v}_{h}:=I_{h}\left(\mathbf{v}^{e}\right) \in \mathbf{U}_{h}$, where $I_{h}$ : $H^{1}(\mathcal{O}(\Gamma))^{3} \rightarrow \mathbf{U}_{h}$ is the Clément interpolation operator, with $\mathcal{O}(\Gamma)$ a neighborhood of $\Gamma$ that contains $\Omega_{h}^{\Gamma}$ and of width $\mathcal{O}(h)$. Based on (5.7), approximation properties of $I_{h}\left(\mathbf{v}^{e}\right)$, and (5.10)-5.11) one gets by standard arguments (see, e.g., [35]):

$$
\left\|\mathbf{v}-I_{h}\left(\mathbf{v}^{e}\right)\right\|_{L^{2}}+h\left\|\nabla_{\Gamma}\left(\mathbf{v}-I_{h}\left(\mathbf{v}^{e}\right)\right)\right\|_{L^{2}} \lesssim h\left\|\nabla_{\Gamma} \mathbf{v}\right\|_{L^{2}} .
$$

Due to (5.8), 5.14), $v_{N}=0$ (since $\mathbf{v} \in \mathbf{V}_{T}$ ), 5.7), and (5.11), we have

$$
\begin{aligned}
\left\|\mathbf{v}_{h}\right\|_{U} & =\left\|I_{h}\left(\mathbf{v}^{e}\right)\right\|_{U} \\
5.8 & \simeq\left\|I_{h}\left(\mathbf{v}^{e}\right)_{T}\right\|_{1}+h^{-1}\left\|I_{h}\left(\mathbf{v}^{e}\right)_{N}\right\|_{L^{2}}+h^{-\frac{1}{2}}\left\|I_{h}\left(\mathbf{v}^{e}\right)\right\|_{L^{2}\left(\Omega_{h}^{\Gamma}\right)} \\
v_{N}=0,55.7 & \left.\lesssim h^{-\frac{1}{2}}\left\|I_{h}\left(\mathbf{v}^{e}\right)\right\|_{H^{1}\left(\Omega_{h}^{\Gamma}\right)}+h^{-1} \| I_{h}\left(\mathbf{v}^{e}\right)-\mathbf{v}\right) \cdot \mathbf{n} \|_{L^{2}} \\
& \lesssim h^{-\frac{1}{2}}\left\|\mathbf{v}^{e}\right\|_{H^{1}(\mathcal{O}(\Gamma))}+h^{-1}\left\|I_{h}\left(\mathbf{v}^{e}\right)-\mathbf{v}^{e}\right\|_{L^{2}} \\
& \lesssim h^{-\frac{1}{2}}\left\|\mathbf{v}^{e}\right\|_{H^{1}(\mathcal{O}(\Gamma))}+h^{-\frac{3}{2}}\left\|I_{h}\left(\mathbf{v}^{e}\right)-\mathbf{v}^{e}\right\|_{L^{2}\left(\Omega_{h}^{\Gamma}\right)} \\
& +h^{-\frac{1}{2}}\left\|I_{h}\left(\mathbf{v}^{e}\right)-\mathbf{v}^{e}\right\|_{H^{1}\left(\Omega_{h}^{\Gamma}\right)} \\
5.7 .14 & \lesssim h^{-\frac{1}{2}}\left\|\mathbf{v}^{e}\right\|_{H^{1}(\mathcal{O}(\Gamma))} \\
5.11 & \lesssim\|\mathbf{v}\|_{1} .
\end{aligned}
$$

Hence, we proved

$$
\left\|\mathbf{v}_{h}\right\|_{U} \lesssim\|\mathbf{v}\|_{1}
$$

Now note, that due to 5.14, 5.13, 5.10, we have

$$
\begin{aligned}
b_{T}\left(\mathbf{v}_{h}, q_{h}\right) & =b_{T}\left(\mathbf{v}, q_{h}\right)-b_{T}\left(\mathbf{v}-I_{h}\left(\mathbf{v}^{e}\right), q_{h}\right) \\
& =\left\|q_{h}\right\|_{L^{2}}^{2}-b_{T}\left(\mathbf{v}-I_{h}\left(\mathbf{v}^{e}\right), q_{h}\right) \\
& =\left\|q_{h}\right\|_{L^{2}}^{2}+\left(\mathbf{v}-I_{h}\left(\mathbf{v}^{e}\right), \nabla_{\Gamma} q_{h}\right)_{L^{2}} \\
& \geq\left\|q_{h}\right\|_{L^{2}}^{2}-\left\|\mathbf{v}-I_{h}\left(\mathbf{v}^{e}\right)\right\|_{L^{2}}\left\|\nabla_{\Gamma} q_{h}\right\|_{L^{2}} \\
& \geq\left\|q_{h}\right\|_{L^{2}}^{2}-c h\|\mathbf{v}\|_{1}\left\|\nabla_{\Gamma} q_{h}\right\|_{L^{2}} \\
& \geq\left\|q_{h}\right\|_{L^{2}}^{2}-c h^{\frac{1}{2}}\|\mathbf{v}\|_{1}\left\|\nabla q_{h}\right\|_{L^{2}\left(\Omega_{h}^{\Gamma}\right)} .
\end{aligned}
$$


Dividing both sides by $\|\mathbf{v}\|_{1}$ and using the results in (5.13) and 5.15) we get, for suitable constants $\tilde{c}_{0}>0$ and $\tilde{c}$ :

$$
\frac{b_{T}\left(\mathbf{v}_{h}, q_{h}\right)}{\left\|\mathbf{v}_{h}\right\|_{U}} \geq \tilde{c}_{0}\left\|q_{h}\right\|_{L^{2}}-\tilde{c} h^{\frac{1}{2}}\left\|\nabla q_{h}\right\|_{L^{2}\left(\Omega_{h}^{\Gamma}\right)} .
$$

Hence, for $c_{p} \geq \tilde{c}^{2}$ we obtain the estimate 5.12.

ASSUMPTiOn 5.1. In the remainder we assume that $c_{p}$ in 4.5 is taken suffciently large such that the discrete inf-sup estimate 5.12 holds.

5.3. Consistency. For the error analysis it is convenient to introduce the bilinear form

$$
\mathbf{A}_{h}((\mathbf{u}, p),(\mathbf{v}, q)):=A_{h}(\mathbf{u}, \mathbf{v})+b_{T}(\mathbf{v}, p)+b_{T}(\mathbf{u}, q)-s_{h}(p, q),
$$

for $(\mathbf{u}, p),(\mathbf{v}, q) \in \mathbf{U} \times Q$. Then, the discrete problem 4.4 has the compact representation: Determine $\left(\mathbf{u}_{h}, p_{h}\right) \in \mathbf{U}_{h} \times Q_{h}$ such that

$$
\mathbf{A}_{h}\left(\left(\mathbf{u}_{h}, p_{p}\right),\left(\mathbf{v}_{h}, q_{h}\right)\right)=\left(\mathbf{f}, \mathbf{v}_{h}\right)_{L^{2}}+\left(g, q_{h}\right)_{L^{2}} \quad \text { for all }\left(\mathbf{v}_{h}, q_{h}\right) \in \mathbf{U}_{h} \times Q_{h} .
$$

Due to 5.12 discrete problem 5.17 has a unique solution, which is denoted by $\left(\mathbf{u}_{h}, p_{h}\right)$. Below we derive a consistency relation of the unique solution $\left(\mathbf{u}_{T}^{*}, p^{*}\right)$ of 2.10)-2.11. To this purpose, we need the normal extensions $\left(\mathbf{u}_{T}^{*}\right)^{e}$ and $\left(p^{*}\right)^{e}$ of $\mathbf{u}_{T}^{*}$ and $p^{*}$, respectively. To simplify the notation these extensions are also denoted by $\mathbf{u}_{T}^{*}$ and $p^{*}$.

LEMma 5.2. Let $\left(\mathbf{u}_{T}^{*}, p^{*}\right)$ be the unique solution of 2.10)-2.11 and $\left(\mathbf{u}_{h}, p_{h}\right)$ the unique solution of 5.17). The following relations hold:

$$
\left.\mathbf{A}_{h}\left(\left(\mathbf{u}_{T}^{*}, p^{*}\right)\right),(\mathbf{v}, q)\right)=(\mathbf{f}, \mathbf{v})_{L^{2}}+(g, q)_{L^{2}}+\int_{\Gamma} E_{s}\left(\mathbf{u}_{T}^{*}\right): v_{N} \mathbf{H} d s-s_{h}\left(p^{*}, q\right)
$$

for all $(\mathbf{v}, q) \in \mathbf{U} \times Q$;

$$
\left.\mathbf{A}_{h}\left(\left(\mathbf{u}_{T}^{*}-\mathbf{u}_{h}, p^{*}-p_{h}\right)\right),\left(\mathbf{v}_{h}, q_{h}\right)\right)=\int_{\Gamma} E_{s}\left(\mathbf{u}_{T}^{*}\right):\left(\mathbf{n} \cdot \mathbf{v}_{h}\right) \mathbf{H} d s-s_{h}\left(p^{*}, q_{h}\right)
$$

for all $\left(\mathbf{v}_{h}, q_{h}\right) \in \mathbf{U}_{h} \times Q_{h}$.

Proof. Using $\nabla \mathbf{u}_{T}^{*} \mathbf{n}=\nabla\left(\mathbf{u}_{T}^{*}\right)^{e} \mathbf{n}=0$ and $u_{N}^{*}=0$ we get

$$
A_{h}\left(\mathbf{u}_{T}^{*}, \mathbf{v}\right)=a_{\tau}\left(\mathbf{u}_{T}^{*}, \mathbf{v}\right)=a\left(\mathbf{u}_{T}^{*}, \mathbf{v}_{T}\right)+\int_{\Gamma} E_{s}\left(\mathbf{u}_{T}^{*}\right): v_{N} \mathbf{H} d s .
$$

Combining this with $b_{T}(\mathbf{v}, p)=b_{T}\left(\mathbf{v}_{T}, p\right), \mathbf{f} \cdot \mathbf{n}=0$, and 2.10)-2.11 we obtain

$$
A_{h}\left(\mathbf{u}_{T}^{*}, \mathbf{v}\right)+b_{T}\left(\mathbf{v}, p^{*}\right)+b_{T}\left(\mathbf{u}_{T}^{*}, q\right)=(\mathbf{f}, \mathbf{v})_{L^{2}}+(g, q)_{L^{2}}+\int_{\Gamma} E_{s}\left(\mathbf{u}_{T}^{*}\right): v_{N} \mathbf{H} d s
$$

for all $(\mathbf{v}, q) \in U \times Q$, which using definition (5.16) yields (5.18). The relation in 5.19 directly folows from 5.18 and 5.17 .

The consistency relation 5.19 describes the violation of the Galerkin orthogonality, due to the stabilization term $s_{h}(\cdot, \cdot)$ and due to the fact that the finite element space contains no-tangential test functions. Below we derive bounds for the consistency error terms in the right-hand side of (5.19). 
5.4. Discretization error bound. We apply the standard theory of saddle point problems to derive the error estimates in the energy norm, defined by

$$
\|\mathbf{u}, p\|:=\left(\|\mathbf{u}\|_{\mathbf{U}}^{2}+\|p\|_{Q}^{2}\right)^{\frac{1}{2}}
$$

Using (5.3)-5.5) one easily checks that $\mathbf{A}_{h}(\cdot, \cdot)$ is continuous on $\mathbf{U} \times Q$ with respect to this product norm. From Lemma 5.1 and definition 5.16 we get the following inf-sup stability result:

$$
0<c_{0} \leq \inf _{\left(\mathbf{v}_{h}, q_{h}\right) \in \mathbf{U}_{h} \times Q_{h}} \sup _{\left(\mathbf{v}_{h}, q_{h}\right) \in \mathbf{U}_{h} \times Q_{h}} \frac{\mathbf{A}_{h}\left(\left(\mathbf{u}_{h}, p_{h}\right),\left(\mathbf{v}_{h}, q_{h}\right)\right)}{\left\|\mathbf{u}_{h}, p_{h}\right\|\left\|\mathbf{v}_{h}, q_{h}\right\|} .
$$

The proof of (5.20) for $s_{h}(\cdot, \cdot)=0$ is given in many finite element textbooks, e.g. 14]. The arguments have straightfoward extensions to the case $s_{h} \neq 0$, cf., for example, [19. From the discrete inf-sup property of the $\mathbf{A}_{h}$ bilinear form and continuity we conclude well-posedness and a stability bound:

$$
\left\|\mathbf{u}_{h}, p_{h}\right\| \lesssim\|\mathbf{f}\|_{L^{2}}+\|g\|_{L^{2}} .
$$

Furthermore, we obtain the following optimal discretization error bound.

THEOREM 5.3. Let $\left(\mathbf{u}_{T}^{*}, p^{*}\right)$ be the solution of (2.10)-(2.11) and assume that $\left(\mathbf{u}_{T}^{*}, p^{*}\right) \in H^{2}(\Gamma) \times H^{1}(\Gamma)$. Let $\left(\mathbf{u}_{h}, p_{h}\right) \in \mathbf{U}_{h} \times Q_{h}$ be the solution of (4.4). The following discretization error bounds hold:

$$
\left\|\mathbf{u}_{T}^{*}-\mathbf{u}_{h}, p^{*}-p_{h}\right\| \lesssim h\left(\left\|\mathbf{u}_{T}^{*}\right\|_{2}+\left\|p^{*}\right\|_{1}\right) .
$$

Here $\|\cdot\|_{2}$ denotes the $H^{2}(\Gamma)$ Sobolev norm.

Proof. Using the stability and consistency properties in 5.20 and (5.19), we obtain, for arbitrary $\left(\mathbf{w}_{h}, \xi_{h}\right) \in \mathbf{U}_{h} \times Q_{h}$ :

$$
\begin{aligned}
& \left\|\mathbf{u}_{h}-\mathbf{w}_{h}, p_{h}-\xi_{h}\right\| \lesssim \sup _{\left(\mathbf{v}_{h}, q_{h}\right) \in \mathbf{U}_{h} \times Q_{h}} \frac{\mathbf{A}_{h}\left(\left(\mathbf{u}_{h}-\mathbf{w}_{h}, p_{h}-\xi_{h}\right),\left(\mathbf{v}_{h}, q_{h}\right)\right)}{\left\|\mathbf{v}_{h}, q_{h}\right\|} \\
& =\sup _{\left(\mathbf{v}_{h}, q_{h}\right) \in \mathbf{U}_{h} \times Q_{h}} \frac{\mathbf{A}_{h}\left(\left(\mathbf{u}_{T}^{*}-\mathbf{w}_{h}, p^{*}-\xi_{h}\right),\left(\mathbf{v}_{h}, q_{h}\right)\right)-s_{h}\left(p^{*}, q_{h}\right)+\int_{\Gamma} E_{s}\left(\mathbf{u}_{T}^{*}\right):\left(\mathbf{n} \cdot \mathbf{v}_{h}\right) \mathbf{H} d s}{\left\|\mathbf{v}_{h}, q_{h}\right\|} \\
& \lesssim\left\|\mathbf{u}_{T}^{*}-\mathbf{w}_{h}, p^{*}-\xi_{h}\right\|+h^{\frac{1}{2}}\left\|\nabla p^{*}\right\|_{L^{2}\left(\Omega_{h}^{\Gamma}\right)}+\tau^{-\frac{1}{2}}\left\|E_{s}\left(\mathbf{u}_{T}^{*}\right)\right\|_{L^{2}} .
\end{aligned}
$$

Hence, using the triangle inequality and $\tau=O\left(h^{-2}\right)$ we get the error bound

$$
\begin{aligned}
& \left\|\mathbf{u}_{T}^{*}-\mathbf{u}_{h}, p^{*}-p_{h}\right\| \\
& \lesssim \inf _{\left(\mathbf{v}_{h}, q_{h}\right) \in \mathbf{U}_{h} \times Q_{h}}\left(\left\|\mathbf{u}_{T}^{*}-\mathbf{v}_{h}, p^{*}-q_{h}\right\|\right)+h^{\frac{1}{2}}\left\|\nabla p^{*}\right\|_{L^{2}\left(\Omega_{h}^{\Gamma}\right)}+h\left\|\mathbf{u}_{T}^{*}\right\|_{1} .
\end{aligned}
$$

Thanks to the norm equivalences in 5.10) (recall that $\left.\nabla p^{*}=\nabla\left(p^{*}\right)^{e}\right)$, we have

$$
h^{\frac{1}{2}}\left\|\nabla p^{*}\right\|_{L^{2}\left(\Omega_{h}^{\Gamma}\right)} \simeq h\left\|\nabla_{\Gamma} p^{*}\right\|_{L^{2}}
$$

For $\left(\mathbf{v}_{h}, \mu_{h}\right) \in \mathbf{U}_{h} \times Q_{h}$ we take optimal finite element (nodal or Clément) interpolants $\mathbf{v}_{h}=I_{h}\left(\mathbf{u}_{T}^{*}\right)=I_{h}\left(\left(\mathbf{u}_{T}^{*}\right)^{e}\right), q_{h}=I_{h}\left(p^{*}\right)$, and use the notation $\mathbf{e}_{u}:=\mathbf{u}_{T}^{*}-I_{h}\left(\mathbf{u}_{T}^{*}\right)$, $e_{p}:=p^{*}-I_{h}\left(p^{*}\right)$. We thus get

$$
\left\|\mathbf{u}_{T}^{*}-\mathbf{u}_{h}, p^{*}-p_{h}\right\| \lesssim\left\|\mathbf{e}_{u}\right\|_{U}+\left\|e_{p}\right\|_{Q}+h\left\|\nabla_{\Gamma} p\right\|_{L^{2}}+h\left\|\mathbf{u}_{T}^{*}\right\|_{1} .
$$


We consider the error term $\left\|\mathbf{e}_{u}\right\|_{U}$. Using interpolation properties of piecewise linear polynomials and their traces, cf., e.g., [35, we have

$$
\begin{aligned}
\left\|\mathbf{e}_{u}\right\|_{U} & \lesssim\left\|\mathbf{e}_{u}\right\|_{V_{*}}+h^{\frac{1}{2}}\left\|\mathbf{e}_{u}\right\|_{H^{1}\left(\Omega_{h}^{\Gamma}\right)} \lesssim\left\|\mathbf{e}_{u}\right\|_{1}+h^{-1}\left\|\mathbf{e}_{u}\right\|_{L^{2}}+h^{\frac{1}{2}}\left\|\mathbf{e}_{u}\right\|_{H^{1}\left(\Omega_{h}^{\Gamma}\right)} \\
& \lesssim h^{-\frac{1}{2}}\left\|\mathbf{e}_{u}\right\|_{H^{1}\left(\Omega_{h}^{\Gamma}\right)}+h^{\frac{1}{2}}\left\|\mathbf{u}_{T}^{*}\right\|_{H^{2}\left(\Omega_{h}^{\Gamma}\right)}+h^{-\frac{3}{2}}\left\|\mathbf{e}_{u}\right\|_{L^{2}\left(\Omega_{h}^{\Gamma}\right)} \\
& \lesssim h^{\frac{1}{2}}\left\|\mathbf{u}_{T}^{*}\right\|_{H^{2}\left(\Omega_{h}^{\Gamma}\right)} \lesssim h\left\|\mathbf{u}_{T}^{*}\right\|_{H^{2}(\Gamma)} .
\end{aligned}
$$

Using similar arguments one derives the bound

$$
\left\|e_{p}\right\|_{Q} \lesssim\left\|e_{p}\right\|_{L^{2}}+h^{\frac{1}{2}}\left\|e_{p}\right\|_{H^{1}\left(\Omega_{h}^{\Gamma}\right)} \lesssim h^{\frac{1}{2}}\left\|p^{*}\right\|_{H^{1}\left(\Omega_{h}^{\Gamma}\right)} \lesssim h\left\|p^{*}\right\|_{H^{1}(\Gamma)} .
$$

The combination of these estimates yields the desired result. $\square$

Corollary 5.4. Let $\left(\mathbf{u}_{T}^{*}, p^{*}\right)$ and $\left(\mathbf{u}_{h}, p_{h}\right)$ be as in Theorem 5.3. The following discretization error estimates hold:

$$
\begin{aligned}
\left\|\mathbf{u}_{T}^{*}-\left(\mathbf{u}_{h}\right)_{T}\right\|_{1}+\left\|p^{*}-p_{h}\right\|_{L^{2}} & \lesssim h\left(\left\|\mathbf{u}_{T}^{*}\right\|_{2}+\left\|p^{*}\right\|_{1}\right), \\
\left\|\mathbf{u}_{h} \cdot \mathbf{n}\right\|_{L^{2}} & \lesssim h^{2}\left(\left\|\mathbf{u}_{T}^{*}\right\|_{2}+\left\|p^{*}\right\|_{1}\right) .
\end{aligned}
$$

Proof. Note that for $\mathbf{v} \in U$ we have $\|\mathbf{v}\|_{U}^{2}=A_{h}(\mathbf{v}, \mathbf{v}) \geq a_{\tau}(\mathbf{v}, \mathbf{v}) \geq\left\|\mathbf{v}_{T}\right\|_{1}^{2}$, and for $q \in Q$ we have $\|q\|_{Q} \geq\|q\|_{L^{2}}$. Using these estimates and the result in (5.22) we obtain (5.27). We also have $\|\mathbf{v}\|_{U}^{2}=A_{h}(\mathbf{v}, \mathbf{v}) \geq a_{\tau}(\mathbf{v}, \mathbf{v}) \geq \tau\left\|v_{N}\right\|_{L^{2}}^{2}$. Combining this with $\tau=c_{\tau} h^{-2}$ and the result in (5.22) we obtain the bound (5.28).

5.5. $L^{2}$-error bound. In this section we use a duality argument to derive an optimal $L^{2}$-norm discretization error bound, based on a regularity assumption for the problem 2.2)-2.3. We assume that the solution $\left(\mathbf{u}=\mathbf{u}_{T}, p\right)$ of the surface Stokes problem (2.2)-2.3) satisfies the regularity estimate:

$$
\left\|\mathbf{u}_{T}\right\|_{2}+\|p\|_{1} \lesssim\|\mathbf{f}\|_{L^{2}}
$$

for any $\mathbf{f} \in L^{2}(\Gamma)^{3}, \mathbf{f} \cdot \mathbf{n}=0$, and $g=0$. Again $\|\cdot\|_{2}$ denotes the $H^{2}(\Gamma)$ Sobolev norm.

THEOREM 5.5. Let $\left(\mathbf{u}_{T}^{*}, p^{*}\right)$ be the solution of 2.10)-2.11) and assume that $\left(\mathbf{u}_{T}^{*}, p^{*}\right) \in\left(H^{2}(\Gamma)\right)^{3} \times H^{1}(\Gamma)$. Let $\left(\mathbf{u}_{h}, p_{h}\right) \in \mathbf{U}_{h} \times Q_{h}$ be the solution of 4.4. The following error estimate holds:

$$
\left\|\mathbf{u}_{T}^{*}-\left(\mathbf{u}_{h}\right)_{T}\right\|_{L^{2}} \lesssim h^{2}\left(\left\|\mathbf{u}_{T}^{*}\right\|_{2}+\left\|p^{*}\right\|_{1}\right)
$$

Proof. We consider 2.10 2.11 with $\mathbf{f}:=\mathbf{u}_{T}^{*}-\left(\mathbf{u}_{h}\right)_{T}=: \mathbf{e}_{h, T}$ and $g=0$. Note that $\mathbf{f} \cdot \mathbf{n}=0$ on $\Gamma$. The unique solution of this problem is denoted by $\left(\mathbf{w}_{T}^{*}, r^{*}\right) \in$ $\mathbf{V}_{T} \times L_{0}^{2}(\Gamma)$. Due to the regularity assumption the $\mathbf{V}_{T} \times L_{0}^{2}(\Gamma)$ pair solves also 2.2 2.3 , and we have

$$
\left\|\mathbf{w}_{T}^{*}\right\|_{2}+\left\|r^{*}\right\|_{1} \lesssim\left\|\mathbf{e}_{h, T}\right\|_{L^{2}}
$$

The normal extensions of the solution pair are also denoted by $\mathbf{w}_{T}^{*}=\left(\mathbf{w}_{T}^{*}\right)^{e}, r^{*}=$ $\left(r^{*}\right)^{e}$. The consistency property 5.18 yields

$\mathbf{A}_{h}\left(\left(\mathbf{w}_{T}^{*}, r^{*}\right),(\mathbf{v}, q)\right)=\left(\mathbf{e}_{h, T}, \mathbf{v}\right)_{L^{2}}+\int_{\Gamma} E_{s}\left(\mathbf{w}_{T}^{*}\right): v_{N} \mathbf{H} d s-s_{h}\left(r^{*}, q\right) \quad \forall(\mathbf{v}, q) \in \mathbf{U} \times Q$. 
Note that the bilinear form $\mathbf{A}_{h}(\cdot, \cdot)$ is symmetric. We take $(\mathbf{v}, q)=\left(\mathbf{u}_{T}^{*}-\mathbf{u}_{h}, p^{*}-p_{h}\right) \in$ $\mathbf{U} \times Q$, which in combination with 5.19 yields

$$
\begin{aligned}
&\left\|\mathbf{e}_{h, T}\right\|_{L^{2}}^{2}=\left(\mathbf{e}_{h, T}, \mathbf{u}_{T}^{*}-\mathbf{u}_{h}\right)_{L^{2}} \\
&= \mathbf{A}_{h}\left(\left(\mathbf{w}_{T}^{*}, r^{*}\right),\left(\mathbf{u}_{T}^{*}-\mathbf{u}_{h}, p^{*}-p_{h}\right)\right)-\int_{\Gamma} E_{s}\left(\mathbf{w}_{T}^{*}\right): \mathbf{H}\left(\mathbf{n} \cdot\left(\mathbf{u}_{T}^{*}-\mathbf{u}_{h}\right)\right) d s \\
&+s_{h}\left(r^{*}, p^{*}-p_{h}\right) \\
&= \mathbf{A}_{h}\left(\left(\mathbf{u}_{T}^{*}-\mathbf{u}_{h}, p^{*}-p_{h}\right),\left(\mathbf{w}_{T}^{*}, r^{*}\right)\right)+\int_{\Gamma} E_{s}\left(\mathbf{w}_{T}^{*}\right): \mathbf{H}\left(\mathbf{n} \cdot \mathbf{u}_{h}\right) d s+s_{h}\left(r^{*}, p^{*}-p_{h}\right) \\
&= \mathbf{A}_{h}\left(\left(\mathbf{u}_{T}^{*}-\mathbf{u}_{h}, p^{*}-p_{h}\right),\left(\mathbf{w}_{T}^{*}-\mathbf{w}_{h}, r^{*}-r_{h}\right)\right) \\
&+\int_{\Gamma} E_{s}\left(\mathbf{w}_{T}^{*}\right):\left(\mathbf{n} \cdot \mathbf{u}_{h}\right) \mathbf{H} d s+\int_{\Gamma} E_{s}\left(\mathbf{u}_{T}^{*}\right):\left(\mathbf{n} \cdot \mathbf{w}_{h}\right) \mathbf{H} d s \\
&+s_{h}\left(r^{*}, p^{*}-p_{h}\right)-s_{h}\left(p^{*}, r_{h}\right),
\end{aligned}
$$

with $\mathbf{w}_{h}:=I_{h}\left(\mathbf{w}_{T}^{*}\right) \in \mathbf{U}_{h}, r_{h}:=I_{h}\left(r^{*}\right) \in Q_{h}$ optimal piecewise linear interpolations of the extended solution $\left(\mathbf{w}_{T}^{*}\right)^{e},\left(r^{*}\right)^{e}$. We consider the terms in (5.32)-(5.34). For the term in 5.32 we use continuity of $\mathbf{A}_{h}(\cdot, \cdot)$, the discretization error bound (5.22), interpolation error bounds as in (5.25), (5.26), and the regularity estimates (5.29), 5.31):

$$
\begin{aligned}
& \left|\mathbf{A}_{h}\left(\left(\mathbf{u}_{T}^{*}-\mathbf{u}_{h}, p^{*}-p_{h}\right),\left(\mathbf{w}_{T}^{*}-\mathbf{w}_{h}, r^{*}-r_{h}\right)\right)\right| \\
& \leq 2\left(\left\|\mathbf{u}_{T}^{*}-\mathbf{u}_{h}\right\|_{U}+\left\|p^{*}-p_{h}\right\|_{Q}\right)\left(\left\|\mathbf{w}_{T}^{*}-\mathbf{w}_{h}\right\|_{U}+\left\|r^{*}-r_{h}\right\|_{Q}\right) \\
& \lesssim h^{2}\left(\left\|\mathbf{u}_{T}^{*}\right\|_{2}+\left\|p^{*}\right\|_{1}\right)\left\|\mathbf{e}_{h, T}\right\|_{L^{2}} .
\end{aligned}
$$

For the term in (5.33) we introduce $\mathbf{e}_{w}:=\mathbf{w}_{T}^{*}-\mathbf{w}_{h}$. Using $\mathbf{n} \cdot \mathbf{w}_{h}=-\mathbf{n} \cdot \mathbf{e}_{w}$, the discretization error bound (5.28), interpolation error bounds as in 5.25), and the regularity estimates 5.29), 5.31, we get:

$$
\begin{aligned}
& \left|\int_{\Gamma} E_{s}\left(\mathbf{w}_{T}^{*}\right):\left(\mathbf{n} \cdot \mathbf{u}_{h}\right) \mathbf{H} d s+\int_{\Gamma} E_{s}\left(\mathbf{u}_{T}^{*}\right):\left(\mathbf{n} \cdot \mathbf{w}_{h}\right) \mathbf{H} d s\right| \\
& \lesssim\left\|\mathbf{w}_{T}^{*}\right\|_{1}\left\|\mathbf{n} \cdot \mathbf{u}_{h}\right\|_{L^{2}}+\left\|\mathbf{u}_{T}^{*}\right\|_{1}\left\|\mathbf{e}_{w}\right\|_{L^{2}} \lesssim h^{2}\left(\left\|\mathbf{u}_{T}^{*}\right\|_{2}+\left\|p^{*}\right\|_{1}\right)\left\|\mathbf{e}_{h, T}\right\|_{L^{2}} .
\end{aligned}
$$

For the term in (5.34) we use the estimates $(5.10)$, the $H^{1}$-boundedness of the interpolation operator $I_{h}$, the discretization error bound $(5.22)$, and the regularity estimates 5.29, 5.31):

$$
\begin{aligned}
& \left|s_{h}\left(r^{*}, p^{*}-p_{h}\right)-s_{h}\left(p^{*}, r_{h}\right)\right| \\
& \lesssim h\left\|\nabla r^{*}\right\|_{L^{2}\left(\Omega_{h}^{\Gamma}\right)}\left\|\nabla\left(p^{*}-p_{h}\right)\right\|_{L^{2}\left(\Omega_{h}^{\Gamma}\right)}+h\left\|\nabla p^{*}\right\|_{L^{2}\left(\Omega_{h}^{\Gamma}\right)}\left\|\nabla I_{h}\left(r^{*}\right)\right\|_{L^{2}\left(\Omega_{h}^{\Gamma}\right)} \\
& \lesssim h\left\|\nabla_{\Gamma} r^{*}\right\|_{L^{2}}\left\|p^{*}-p_{h}\right\|_{Q}+h^{\frac{3}{2}}\left\|\nabla_{\Gamma} p^{*}\right\|_{L^{2}}\left\|r^{*}\right\|_{H^{1}\left(\Omega_{h}^{\Gamma}\right)} \\
& \lesssim h^{2}\left(\left\|\mathbf{u}_{T}^{*}\right\|_{2}+\left\|p^{*}\right\|_{1}\right)\left\|\mathbf{e}_{h, T}\right\|_{L^{2}}+h^{2}\left\|p^{*}\right\|_{1}\left\|r^{*}\right\|_{1} \\
& \lesssim h^{2}\left(\left\|\mathbf{u}_{T}^{*}\right\|_{2}+\left\|p^{*}\right\|_{1}\right)\left\|\mathbf{e}_{h, T}\right\|_{L^{2}} .
\end{aligned}
$$

Using estimates 5.35, 5.36, 5.37) in 5.32, 5.33, and 5.34 we obtain error bound 5.30.

6. Condition number estimate and algebraic solver. It is well-known [29, 7. that for unfitted finite element methods there is an issue concerning algebraic stability. In fact, the matrices that represent the discrete problem may have very bad 
conditioning due to small cuts in the geometry. One way to remedy this stability problem is by using stabilization methods. See, e.g., [7, 31]. In this section we show that the 'volume normal derivative' stabilizations in the bilinear forms $A_{h}(\cdot, \cdot)$ in (4.1) and $s_{h}(\cdot, \cdot)$ in 4.2), with scaling as in 4.5), remove any possible algebraic instability. More precisely, we show that the condition number of the stiffness matrix corresponding to the saddle point problem (4.4) is bounded by $\mathrm{ch}^{-2}$, where the constant $c$ is independent of the position of the interface. Furthermore, we present an optimal Schur complement preconditioner.

Let integer $n>0, m>0$ be the number of active degrees of freedom in $\mathbf{U}_{h}$ and $Q_{h}$ spaces, i.e., $n=\operatorname{dim}\left(\mathbf{U}_{h}\right), m=\operatorname{dim}\left(M_{h}\right)$, and $P_{h}^{Q}: \mathbb{R}^{n} \rightarrow \mathbf{U}_{h}$ and $P_{h}^{Q}: \mathbb{R}^{m} \rightarrow Q_{h}$ are canonical mappings between the vectors of nodal values and finite element functions. Denote by $\langle\cdot, \cdot\rangle$ and $\|\cdot\|$ the Euclidean scalar product and the norm. For matrices, $\|\cdot\|$ denotes the spectral norm in this section.

Let us introduce several matrices. Let $A, M_{u} \in \mathbb{R}^{n \times n}, B \in \mathbb{R}^{n \times m}, C, M_{p} \in \mathbb{R}^{m \times m}$ be such that

$$
\begin{aligned}
\langle A \vec{u}, \vec{v}\rangle & =A_{h}\left(P_{h}^{U} \vec{u}, P_{h}^{U} \vec{v}\right),\langle B \vec{u}, \vec{\lambda}\rangle=b_{T}\left(P_{h}^{U} \vec{u}, P_{h}^{Q} \vec{\lambda}\right),\langle C \vec{\lambda}, \vec{\mu}\rangle=s_{h}\left(P_{h}^{Q} \vec{\lambda}, P_{h}^{Q} \vec{\mu}\right), \\
\left\langle M_{u} \vec{u}, \vec{v}\right\rangle & =\left(P_{h}^{U} \vec{u}, P_{h}^{U} \vec{v}\right)_{L^{2}\left(\Omega_{h}^{\Gamma}\right)}, \quad\left\langle M_{p} \vec{\lambda}, \vec{\mu}\right\rangle=\left(P_{h}^{Q} \vec{\lambda}, P_{h}^{Q} \vec{\mu}\right)_{L^{2}\left(\Omega_{h}^{\Gamma}\right)}, \\
\left\langle S_{Q} \vec{\lambda}, \vec{\mu}\right\rangle & =\left(P_{h}^{Q} \vec{\lambda}, P_{h}^{Q} \vec{\mu}\right)_{L^{2}}+h\left(\nabla\left(P_{h}^{Q} \vec{\lambda}\right), \nabla\left(P_{h}^{Q} \vec{\mu}\right)\right)_{L^{2}\left(\Omega_{h}^{\Gamma}\right)}
\end{aligned}
$$

for all $\vec{u}, \vec{v} \in \mathbb{R}^{n}, \quad \vec{\mu}, \vec{\lambda} \in \mathbb{R}^{m}$. Note that the mass matrices $M_{u}$ and $M_{p}$ do not depend on how the surface $\Gamma$ intersects the domain $\Omega_{h}^{\Gamma}$. Since the family of background meshes is shape regular, these mass matrices have a spectral condition number that is uniformly bounded, independent of $h$ and of how $\Gamma$ intersects the background triangulation $\mathcal{T}_{h}$. Furthermore, for the symmetric positive definite matrix $S_{Q}$ we have

$$
\left\langle S_{Q} \vec{\lambda}, \vec{\lambda}\right\rangle=\left\|P_{h}^{Q} \vec{\lambda}\right\|_{Q}^{2} \quad \text { for all } \vec{\lambda} \in \mathbb{R}^{m}
$$

cf. 5.2. We also introduce the system matrix and its Schur complement:

$$
\mathcal{A}:=\left[\begin{array}{cc}
A & B^{T} \\
B & -C
\end{array}\right], \quad S:=B A^{-1} B^{T}+C .
$$

The algebraic system resulting from the finite element method 4.4 has the form

$$
\mathcal{A} \vec{x}=\vec{b}, \quad \text { with some } \vec{x}, \vec{b} \in \mathbb{R}^{n+m} .
$$

We will propose a block-diagonal preconditioner of the matrix $\mathcal{A}$. We start by analyzing preconditioners for matrices $A$ and $S$. In the following lemma we make use of spectral inequalities for symmetric matrices.

LEMMA 6.1. There are strictly positive constants $\nu_{A, 1}, \nu_{A, 2}, \nu_{S, 1}, \nu_{S, 2}, \tilde{\nu}_{S, 1}$, $\tilde{\nu}_{S, 2}$, independent of $h$ and of how $\Gamma$ intersects $\mathcal{T}_{h}$ such that the following spectral inequalities hold:

$$
\begin{aligned}
\nu_{A, 1} h^{-1} M_{u} & \leq A \leq \nu_{A, 2} h^{-3} M_{u}, \\
\nu_{S, 1} h^{-1} M_{p} & \leq S \leq \nu_{S, 2} h^{-1} M_{p}, \\
\tilde{\nu}_{S, 1} S_{Q} & \leq S \leq \tilde{\nu}_{S, 2} S_{Q} .
\end{aligned}
$$


Proof. Note that

$$
\frac{\langle A \vec{v}, \vec{v}\rangle}{\left\langle M_{u} \vec{v}, \vec{v}\right\rangle}=\frac{A_{h}\left(P_{h}^{U} \vec{v}, P_{h}^{U} \vec{v}\right)}{\left\|P_{h}^{U} \vec{v}\right\|_{L^{2}\left(\Omega_{h}^{\Gamma}\right)}^{2}} \quad \text { for all } \vec{v} \in \mathbb{R}^{n} .
$$

Let $\mathbf{v}_{h}=P_{h}^{U} \vec{v}$. From 5.8 we get

$$
\nu_{A, 1} h^{-1} \leq \frac{A_{h}\left(\mathbf{v}_{h}, \mathbf{v}_{h}\right)}{\left\|\mathbf{v}_{h}\right\|_{L^{2}\left(\Omega_{h}^{\Gamma}\right)}^{2}} \quad \text { for all } \mathbf{v}_{h} \in \mathbf{U}_{h},
$$

which proves the lower bound in 6.2. For the upper bound we use 5.7 (componentwise) and a finite element inverse estimate,

$$
\begin{aligned}
& \left\|\left(\mathbf{v}_{h}\right)_{T}\right\|_{1}^{2}+h^{-2}\left\|\left(\mathbf{v}_{h}\right)_{N}\right\|_{L^{2}}^{2} \leq\left\|\mathbf{v}_{h}\right\|_{1}^{2}+h^{-2}\left\|\mathbf{v}_{h}\right\|_{L^{2}}^{2} \\
& \lesssim h^{-1}\left\|\mathbf{v}_{h}\right\|_{H^{1}\left(\Omega_{h}^{\Gamma}\right)}^{2}+h^{-3}\left\|\mathbf{v}_{h}\right\|_{L^{2}\left(\Omega_{h}^{\Gamma}\right)}^{2} \lesssim h^{-3}\left\|\mathbf{v}_{h}\right\|_{L^{2}\left(\Omega_{h}^{\Gamma}\right)}^{2} \quad \text { for all } \mathbf{v}_{h} \in \mathbf{U}_{h} .
\end{aligned}
$$

Combining this with (5.8), we obtain

$$
\frac{A_{h}\left(\mathbf{v}_{h}, \mathbf{v}_{h}\right)}{\left\|\mathbf{v}_{h}\right\|_{L^{2}\left(\Omega_{h}^{\Gamma}\right)}^{2}} \leq \nu_{A, 2} h^{-3} \text { for all } \mathbf{v}_{h} \in \mathbf{U}_{h},
$$

for a suitable constant $\nu_{A, 2}$. This proves the second inequality in 6.2. For the Schur complement matrix $S=B A^{-1} B^{T}+C$, we first note that

$$
\left\langle B A^{-1} B^{T} \vec{\lambda}, \vec{\lambda}\right\rangle=\left(\sup _{\mathbf{v}_{h} \in \mathbf{U}_{h}} \frac{b_{T}\left(\mathbf{v}_{h}, \mu_{h}\right)}{\left\|\mathbf{v}_{h}\right\|_{U}}\right)^{2}, \quad \mu_{h}:=P_{h}^{Q} \vec{\lambda}
$$

Using the discrete inf-sup estimate 5.12 ) and $(5.9)$, we thus get

$$
\begin{aligned}
\langle S \vec{\lambda}, \vec{\lambda}\rangle & =\left(\sup _{\mathbf{v}_{h} \in \mathbf{U}_{h}} \frac{b_{T}\left(\mathbf{v}_{h}, \mu_{h}\right)}{\left\|\mathbf{v}_{h}\right\|_{U}}\right)^{2}+s_{h}\left(\mu_{h}, \mu_{h}\right) \\
& \gtrsim\left\|\mu_{h}\right\|_{Q}^{2} \gtrsim h^{-1}\left\|\mu_{h}\right\|_{L^{2}\left(\Omega_{h}^{\Gamma}\right)}^{2} \gtrsim h^{-1}\left\langle M_{p} \vec{\lambda}, \vec{\lambda}\right\rangle,
\end{aligned}
$$

which proves the first inequalities in (6.3) and 6.4). Using

$$
\frac{b_{T}\left(\mathbf{v}_{h}, \mu_{h}\right)}{\left\|\mathbf{v}_{h}\right\|_{U}} \leq \frac{\left\|\left(\mathbf{v}_{h}\right)_{T}\right\|_{1}\left\|\mu_{h}\right\|_{L^{2}}}{\left\|\mathbf{v}_{h}\right\|_{U}} \leq\left\|\mu_{h}\right\|_{L^{2}}
$$

and $(5.9)$ we also obtain

$$
\begin{aligned}
\langle S \vec{\lambda}, \vec{\lambda}\rangle & \leq\left\|\mu_{h}\right\|_{L^{2}}^{2}+s_{h}\left(\mu_{h}, \mu_{h}\right) \leq \max \left\{1, c_{p}\right\}\left\|\mu_{h}\right\|_{Q}^{2} \\
& \lesssim h^{-1}\left\|\mu_{h}\right\|_{L^{2}\left(\Omega_{h}^{\Gamma}\right)}^{2} \lesssim h^{-1}\left\langle M_{p} \vec{\lambda}, \vec{\lambda}\right\rangle,
\end{aligned}
$$

which proves the second inequalities in 6.3 and 6.4.

The results in 6.3 and 6.4 yield that both the pressure mass matrix $M_{p}$ and the matrix $S_{Q}$ are optimal preconditioners for the Schur complement matrix $S$. This is an analog of a well-known result for (stabilized) finite element discretizations of the Stokes problem in Euclidean spaces. 
We introduce a block diagonal preconditioner

$$
Q:=\left[\begin{array}{cc}
Q_{A} & 0 \\
0 & Q_{S}
\end{array}\right]
$$

for $\mathcal{A}$. In order to analyze it, we can apply analyses known from the literature, e.g. section 4.2 in 13 .

COROLlaRY 6.2. The following estimate holds for some $c>0$ independent of $h$ and of how $\Gamma$ cuts through the background mesh:

$$
\operatorname{cond}(\mathcal{A})=\|\mathcal{A}\|\left\|\mathcal{A}^{-1}\right\| \leq c h^{-2} .
$$

Proof. Take $Q_{A}:=M_{u}, Q_{S}:=M_{p}$. We can apply Theorem 4.7 from [13] with (notation from [13]) preconditioners $\mathbf{P}=M_{u}, T=M_{p}$. This yields that all eigenvalues of $Q^{-1} \mathcal{A}$ are contained in the union of intervals

$$
\left[-c_{0} h^{-1},-c_{1} h^{-1}\right] \cup\left[d_{0} h^{-1}, d_{1}, h^{-3}\right],
$$

with constants $c_{0}>c_{1}>0,0<d_{0}<d_{1}$ that depend only on the constants $\nu_{A, i}$, $\nu_{S, i}$ in 6.2 , 6.3). From this spectral estimate and the fact that $Q$ has a uniformly bounded condition number we conclude that 6.9 holds.

The application of Theorem 4.7 from 13 also yields the following result.

Corollary 6.3. Let $Q_{A} \sim A$ be a uniformly spectrally equivalent preconditioner of $A$ and $Q_{S}:=M_{p}$ or $Q_{S}:=S_{Q}$. For the spectrum $\sigma\left(Q^{-1} \mathcal{A}\right)$ of the preconditioned matrix we have

$$
\sigma\left(Q^{-1} \mathcal{A}\right) \subset\left(\left[C_{-}, c_{-}\right] \cup\left[c_{+}, C_{+}\right]\right),
$$

with some constants $C_{-}<c_{-}<0<c_{+}<C_{+}$independent of $h$ and the position of $\Gamma$.

In section 7.1 we study the performance of preconditioner 6.8 with $Q_{A}=A$ and $Q_{S}=S_{Q}$.

7. Numerical experiments. A series of numerical tests is presented to showcase the main features and performance of the TraceFEM for the surface Stokes problem.

As explained in Remark 4.1, we approximate the surface $\Gamma$ with a piecewise planar approximation $\Gamma_{h}$, with $\operatorname{dist}\left(\Gamma, \Gamma_{h}\right) \lesssim h^{2}$. We use piecewise linear finite elements for both velocity and pressure in problem (4.4). Higher order finite elements are possible (see, e.g., [16]) and will be addressed in a forthcoming paper.

We remind that a second source of geometric error is given by the approximation $\mathbf{n}_{h}$ of the exact normal $\mathbf{n}$. For the numerical results below, we choose an approximation $\mathbf{n}_{h}=\frac{\nabla \phi_{h}}{\left\|\nabla \phi_{h}\right\|_{2}}$, where where $\phi_{h}$ is defined as a $P_{2}$ nodal interpolant of the level set function.

We first consider the Stokes problem, i.e. 2.2 -2.3 on the unit sphere. To satisfy assumption 2.1. we set $\alpha=1$. The goals of this first test are: to check the spatial accuracy of the TraceFEM, thereby verifying numerically the theoretical results in Corollary 5.4 and Theorem 5.5 , to check the sensitivity of the spatial discretization error with respect to the pressure stabilization parameter $\rho_{p}=c_{p} h$; and to illustrate the behavior of a preconditioned MINRES solver. Regarding other parameters (cf. 
(4.5) we note the following: different choices of the parameter $\rho_{u}$ were analysed in 7, 16, 17, and the TraceFEM was found to be very robust with respect to the variation of this parameter. Based on these experiences we simply set $\rho_{u}:=h$. Regarding the penalty parameter $\tau$, previous numerical studies of vector surface problems [21, 22, 38] all suggest that $\tau$ should be "sufficiently" large. This is consistent with our experience, which shows that the approach is largely insensitive to the variation of $\tau$ once it is large enough.

Next, we consider in section 7.2 the unsteady Stokes problem discretized in time by the backward Euler method. With this second test we want to illustrate the expected evolution of the flow to the reference Killing field for different meshes and different time steps. Finally, in section 7.3 we show a flow field computed on an implicitly given manifold with (strongly) varying curvature.

7.1. Stokes problem on the unit sphere. The surface $\Gamma$ is the unit sphere, centered at the origin. We characterize it as the zero level of the level set function $\phi(\mathbf{x})=\|\mathbf{x}\|_{2}-1$, where $\mathbf{x}=\left(x_{1}, x_{2}, x_{3}\right)^{T}$. We consider the following exact solution to problem (2.2)-(2.3):

$$
\begin{aligned}
& \mathbf{u}^{*}=\mathbf{P}\left(-x_{3}^{2}, x_{2}, x_{1}\right)^{T} \in \mathbf{V}_{T}, \\
& p^{*}=x_{1} x_{2}^{3}+x_{3} \in L_{0}^{2}(\Gamma) .
\end{aligned}
$$

The forcing term $\mathbf{f}$ in eq. 2.2 and source term $g$ in eq. 2.3 are readily computed from the above exact solution. Notice that the pressure average is zero. We set $\alpha=1$ to exclude the Killing vectors on a sphere from the kernel. The sphere is embedded in an outer cubic domain $\Omega=[-5 / 3,5 / 3]^{3}$. The triangulation $\mathcal{T}_{h_{\ell}}$ of $\Omega$ consists of $n_{\ell}^{3}$ sub-cubes, where each of the sub-cubes is further refined into 6 tetrahedra. Here $\ell \in \mathbb{N}$ denotes the level of refinement, with the associated mesh size $h_{\ell}=\frac{10 / 3}{n_{\ell}}$ and $n_{\ell}=2^{\ell+1}$. Parameters $\tau, \rho_{p}$, and $\rho_{u}$ are set as in 4.5 with values of $c_{\tau}, c_{p}$, and $c_{u}$ that will be specified for each case. The velocity and pressure computed with the mesh associated to refinement level $\ell=5$ are illustrated in Figure 7.1.

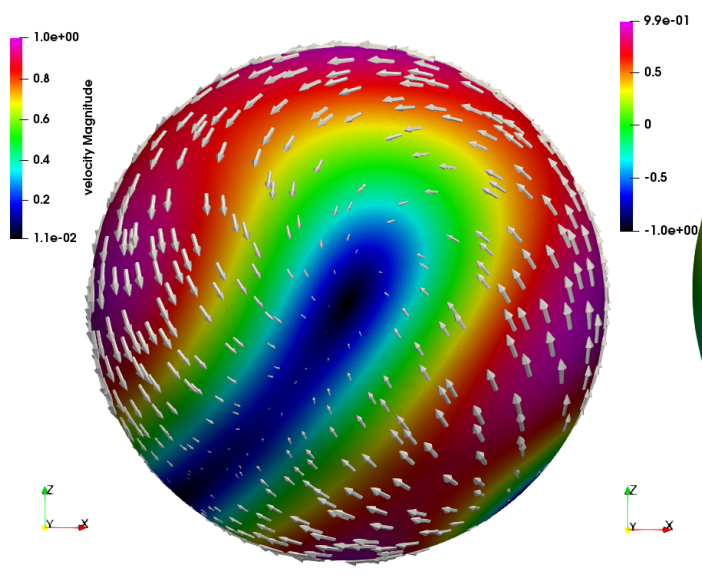

(a) Velocity

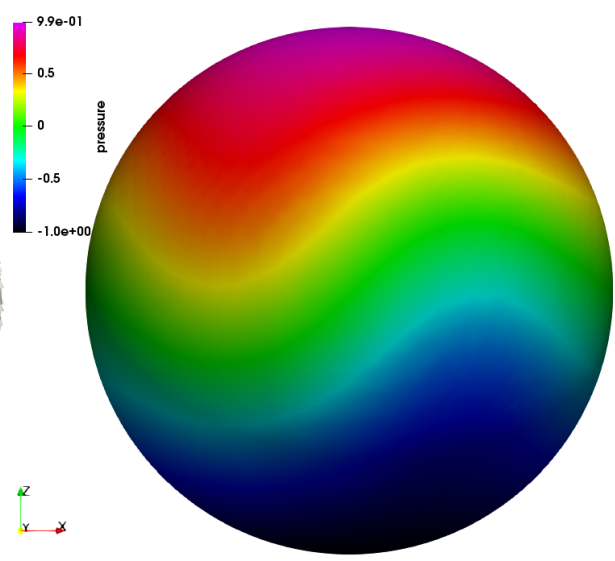

(b) Pressure

Fig. 7.1: Velocity and pressure computed on refinement level $\ell=5$ mesh. 


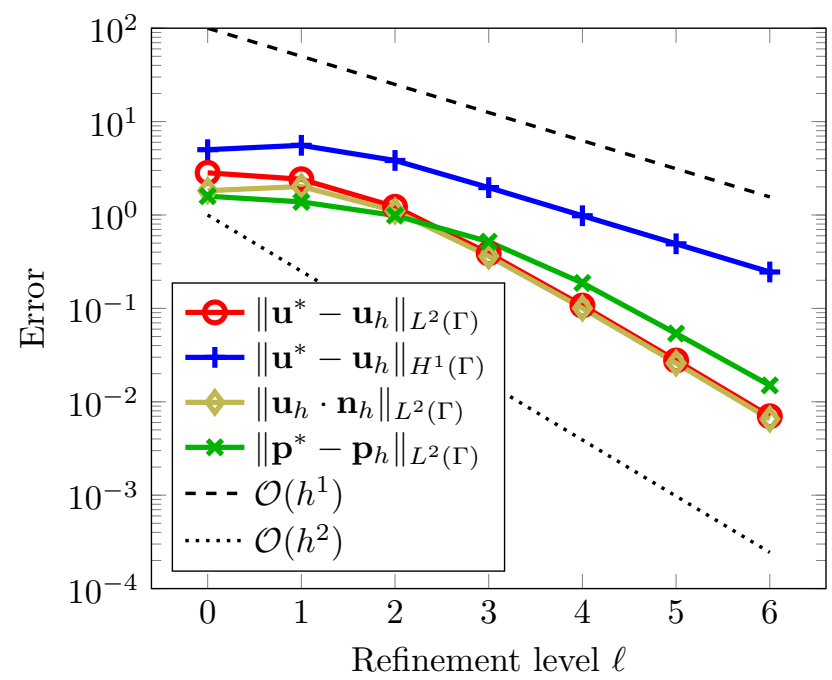

Fig. 7.2: $L^{2}(\Gamma)$ and $H^{1}(\Gamma)$ norms of the error for the velocity, $L^{2}(\Gamma)$ norm of the normal velocity and the $L^{2}(\Gamma)$ norm of the error for the pressure plotted against the refinement level $\ell$. The computational results have been obtained with $\rho_{p}=h$.

We report in Fig. 7.2 the $L^{2}(\Gamma)$ and $H^{1}(\Gamma)$ norms of the error for the velocity, $L^{2}(\Gamma)$ norm of the normal velocity component, and the $L^{2}(\Gamma)$ norm of the error for the pressure plotted against the refinement level $\ell$. All the norms reported in Fig. 7.2 . and also in Fig. 7.3 and 7.5 , are computed on the approximate surface $\Gamma_{h}$, while for the exact velocity and pressure solutions (on $\Gamma$ ) we use the continuous extension as specified in 7.1). We observe that optimal convergence orders are achieved for $\| \mathbf{u}^{*}-$ $\mathbf{u}_{h} \|_{H^{1}(\Gamma)}$ and $\left\|\mathbf{u}^{*}-\mathbf{P} \mathbf{u}_{h}\right\|_{L^{2}(\Gamma)}$, as predicted by the theoretical results in Corollary 5.4 and Theorem 5.5. We note though that the theoretical analysis does not account for the geometric errors. For $\left\|p-p_{h}\right\|_{L^{2}(\Gamma)}$, we see a faster convergence than predicted by Corollary 5.4. The results shown in Fig. 7.2 have been obtained for $c_{\tau}=1, c_{p}=1$, and $c_{u}=1$.

We now vary the value of parameter $c_{p}$. Figure 7.3 shows the $L^{2}(\Gamma)$ and $H^{1}(\Gamma)$ norms of the error for the velocity and the $L^{2}(\Gamma)$ norm of the error for the pressure for $c_{p} \in\left[5 \cdot 10^{-3}, 10\right]$. We see that as the value of $c_{p}$ moves away from 1 the errors either remain almost constant or slightly increase. Thus among the values of $c_{p}$ that we considered, $c_{p}=1$ is close to an "optimal" choice, and there is a low sensitivity of the accuracy depending on $c_{p}$.

For the solution of the linear system (6.1), we used the preconditioned MINRES method with the block-diagonal preconditioner 6.8). The preconditioner $Q_{A}$ is defined through the application a standard SSOR-preconditioned CG method to solve $A \vec{v}=\vec{b}$ iteratively, with a tolerance such that the initial residual is reduced by a factor of $10^{4}$. The same strategy is used to define $Q_{S}$, i.e. it is also defined through the application a standard SSOR-preconditioned CG method to solve $S_{Q} \vec{p}=\vec{c}$ iteratively, with a tolerance such that the initial residual is reduced by a factor of $10^{4}$. As initial guess for the MINRES method we chose the zero vector. We adopted a stopping criterium based on the Euclidean norm of the residual of system (6.1) and set the stopping tolerance to $10^{-8}$. We report in Tables 7.1 and 7.2 the number of MINRES 


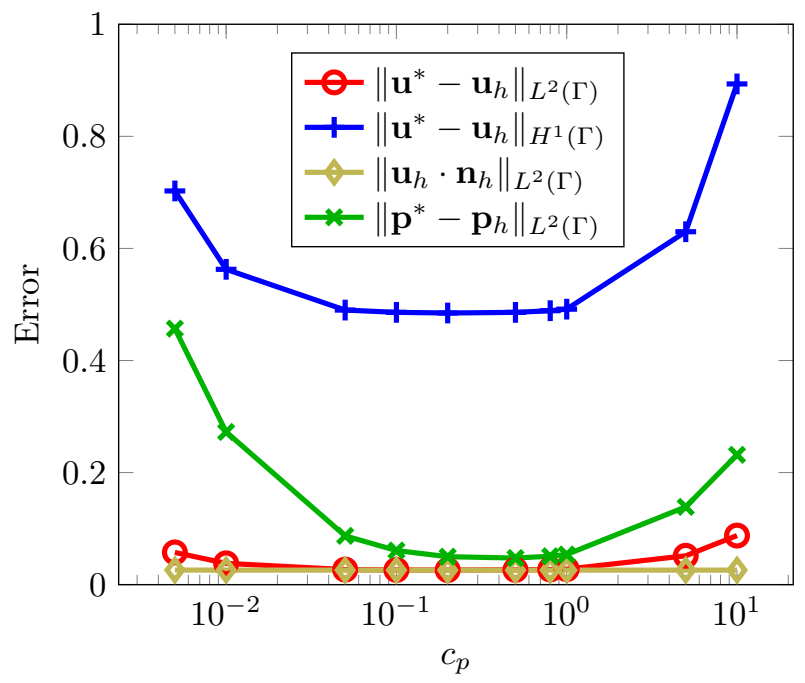

Fig. 7.3: $L^{2}(\Gamma)$ and $H^{1}(\Gamma)$ norms of the error for the velocity, $L^{2}(\Gamma)$ norm of the normal velocity and the $L^{2}(\Gamma)$ norm of the error for the pressure plotted against the value of $c_{p}$. The computational results have been obtained with the $\ell=5$ mesh.

iterations for the simulations in Figures 7.2 and 7.3 , respectively.

\begin{tabular}{r|ccccccc}
\hline$\ell$ & 0 & 1 & 2 & 3 & 4 & 5 & 6 \\
\hline \# iterations & 10 & 14 & 20 & 26 & 29 & 29 & 29 \\
average \# inner CG iterations, precond. $A$ & 5 & 8 & 16 & 27 & 51 & 98 & 184 \\
average \# inner CG iterations, precond. $S_{Q}$ & 6 & 7 & 7 & 8 & 8 & 8 & 8
\end{tabular}

Table 7.1: Total number of MINRES iterations for different refinement levels $\ell$ and the average number of inner CG iterations needed to compute the action of the preconditioners. For all the simulations we set $c_{p}=1$.

\begin{tabular}{r|ccccccc}
\hline$c_{p}$ & 0.01 & 0.05 & 0.1 & 0.5 & 1 & 5 & 10 \\
\hline \# iterations & 120 & 54 & 39 & 20 & 29 & 64 & 86
\end{tabular}

Table 7.2: Total number of MINRES iterations for different values of $c_{p}$. All the simulations used the refinement level $\ell=5$ mesh.

From Table 7.1 we see that the number of MINRES iterations grows from refinement level $\ell=0$ to $\ell=2$, then it increases slightly till $\ell=4$, and for $\ell \geq 4$ it levels off. This observation is consistent with the result of Corollary 6.3. We also report the average number of inner preconditioned CG iterations needed to compute the matrix-vector product with preconditioners $A$ and $S_{Q}$. We see that $S_{Q}$ is uniformly well-conditioned, while the linear growth of the number of the preconditioned CG 
iterations for $A$ is similar to what one expects for a standard FE discretization of the Poisson problem.

From Table 7.2 we observe that when using the mesh associated with $\ell=5$ the number of MINRES iterations attains its minimum approximately for the same value of $c_{p}$ that minimizes the discretization error. For $c_{p} \leq 10^{-3}$, MINRES did not converge within 300 iterations. For example, for $c_{p}=10^{-3}$ (resp., $c_{p}=10^{-4}$ ) the stopping criterion is satisfied after 379 (resp., 1182) iterations. This may be related to the fact that the Schur complement is symmetric and positive definite only if $c_{p}$ is sufficiently large, cf. Lemma 5.1. Hence, the used preconditioner is more sensitive to variations of $c_{p}$ than to mesh refinement.

From the results in this section we deduce that the straightforward choice $c_{\tau}=$ $c_{p}=c_{u}=1$ is a good compromise between minimizing the spatial discretization error and keeping the number of MINRES iterations low. Thus, the results in sections 7.2 and 7.3 below have been obtained with $c_{\tau}=c_{p}=c_{u}=1$.

7.2. Unsteady Stokes problem on the unit sphere. In this section, we consider the unsteady incompressible Stokes problem posed on the surface of a unit sphere and discretized in time with the implicit Euler method. This gives rise to problem as in 2.2 -2.3 with $\alpha=1 / \Delta t$, where $\Delta t$ is a time step. We assume that no external force is applied and we set $g=0$ in equation $(2.3)$. The initial velocity at $t=0$ is chosen to be

$$
\mathbf{u}_{0}=\mathbf{n} \times \nabla_{\Gamma}\left(Y_{1}^{x_{3}}+Y_{1}^{x_{2}}+Y_{2}^{x_{3}}+Y_{3}^{x_{3}}\right),
$$

where $Y_{k}^{x_{l}}$ is a real spherical harmonic of zero order and degree $k$, axisymmetric with respect to the $x_{l}$ coordinate axis. Notice that $\operatorname{div}_{\Gamma} \mathbf{u}_{0}=0$. It can easily be checked that all spherical harmonics have zero angular momentum except for those of first degree. Since there is only one Killing vector field with given total angular momentum, we expect the solution to evolve towards the reference Killing vector field with the same non-zero angular momentum as $\mathbf{u}_{0}$. Therefore, the reference Killing vector field corresponds to the rotating motion over the axis $\mathbf{e}_{3}+\mathbf{e}_{2}$ with angular momentum $\int_{\Gamma} \mathbf{r} \times \mathbf{n} \times \nabla_{\Gamma}\left(Y_{1}^{x_{3}}+Y_{1}^{x_{2}}\right) d S$, where $\mathbf{r}$ is the vector connecting the origin of the axes to point $\mathbf{x}$ lying on the unit sphere.

We consider the meshes associated to refinement levels $\ell=2,3,4,5$ used in Sec.7.1 and two values for the time step $\Delta t=0.1,0.01$. Fig. 7.4 shows both the initial velocity and the reference Killing vector field computed with mesh $\ell=5$ and $\Delta t=0.1$.

Figure 7.5 shows the evolution of the computed kinetic energy over time for refinement levels $\ell=2,3,4,5$. Kinetic energy was calculated after each time step as $0.5\left\|\mathbf{u}_{h}\right\|_{L^{2}(\Gamma)}^{2}$. The reference line corresponds to kinetic energy of the reference Killing vector field.

The time-dependent discrete surface Stokes problem is a dissipative dynamical system. Hence its kinetic energy asymptotically decays exponentially at the rate equal to its minimal eigenvalue. Since the sphere has non-trivial Killing fields, for the original differential problem the asymptotic decay rate is zero. Although this paper does not analyze eigenvalue convergence, one may expect that the FE problem approximates the asymptotic evolution of the system. As an indication for this, we fit the kinetic energy computed with the meshes associated to refinement levels $\ell=2,3,4,5$ and $\Delta t=0.1,0.01$ with the function $A \exp (-\lambda t)$ for time $t \in[2,5]$. We report in Table 7.6 the values of $\lambda$ for each case. We notice that as the time step goes from 0.1 to 0.01 there is only a small difference in the value of $\lambda$. From Table 7.6 we see that as the mesh gets finer the discrete approximations of the asymptotic decay 


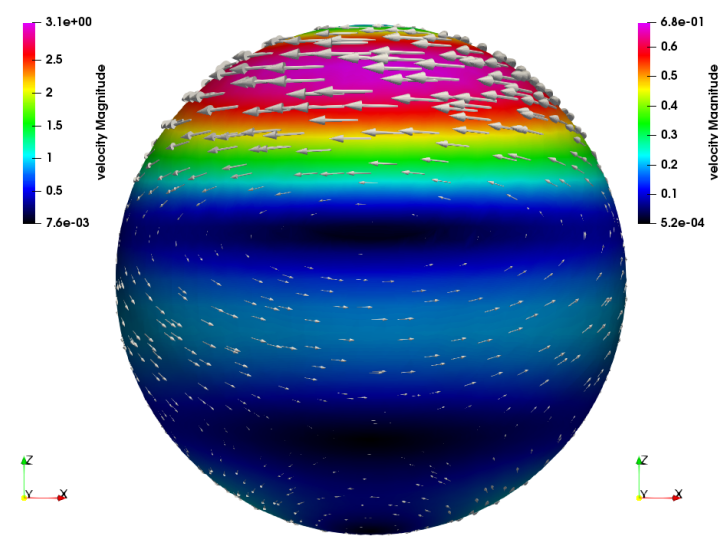

(a) Initial velocity

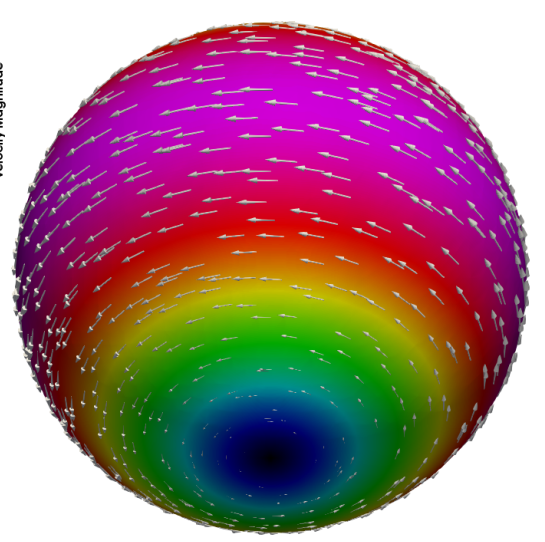

(b) Killing vector field

Fig. 7.4: Initial velocity and reference Killing vector field computed with the mesh $\ell=5$ and $\Delta t=0.1$.
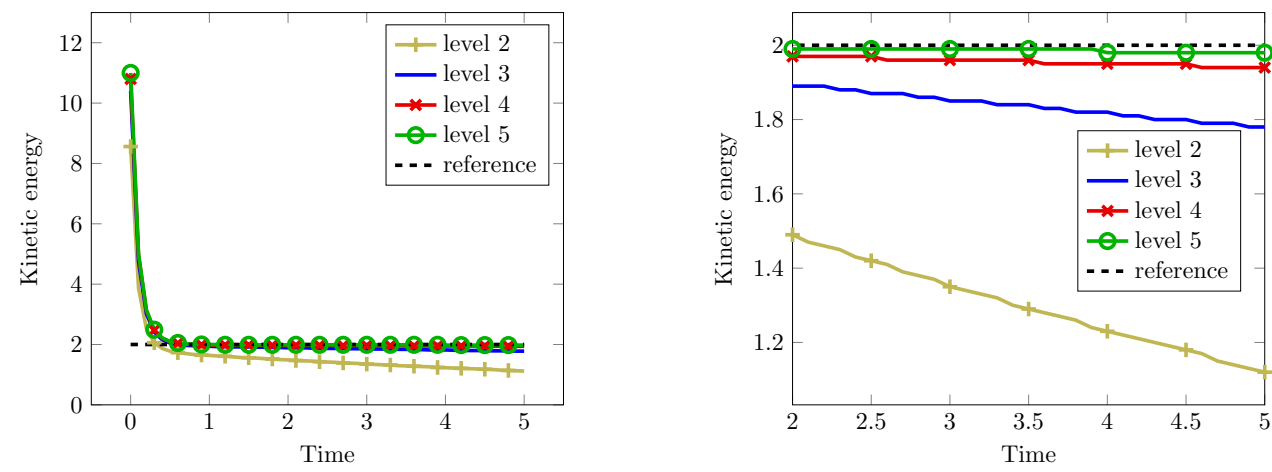

Fig. 7.5: Time evolution of the kinetic energy computed for different refinement levels and $\Delta t=0.1$ (left). Zoom-in for time $t=[2,5] \mathrm{s}$ (right).

rate $\lambda$ converge to zero approximately as $O\left(h^{2}\right)$. This convergence is consistent with the second order accuracy of our finite element method.

7.3. Source and sink flow on an implicitly defined surface. In this section, we consider the unsteady surface Stokes problem posed on a more complex manifold. The surface $\Gamma$ is implicitly defined as the zero level-set of

$$
\begin{aligned}
\phi\left(x_{1}, x_{2}, x_{3}\right)= & \left(x_{1}^{2}+x_{2}^{2}-4\right)^{2}+\left(x_{2}^{2}-1\right)^{2}+\left(x_{2}^{2}+x_{3}^{2}-4\right)^{2}+\left(x_{1}^{2}-1\right)^{2} \\
& +\left(x_{1}^{2}+x_{3}^{2}-4\right)^{2}+\left(x_{3}^{2}-1\right)^{2}-13 .
\end{aligned}
$$

The example of $\Gamma$ is taken from $[9]$. We embed $\Gamma$ in the cube $[-3,3]^{3}$ centered at the origin. We assume that no external force is applied and we start the simulation from fluid at rest, i.e. initial velocity is $\mathbf{u}_{0}=\mathbf{0}$. The flow is driven by the non-zero source 


\begin{tabular}{r|cccccccc}
\hline$\ell$ & 2 & 2 & 3 & 3 & 4 & 4 & 5 & 5 \\
$\Delta t$ & $1 \mathrm{e}-1$ & $1 \mathrm{e}-2$ & $1 \mathrm{e}-1$ & $1 \mathrm{e}-2$ & $1 \mathrm{e}-1$ & $1 \mathrm{e}-2$ & $1 \mathrm{e}-1$ & $1 \mathrm{e}-2$ \\
\hline$\lambda$ & $9.40 \mathrm{e}-2$ & $9.75 \mathrm{e}-2$ & $2.13 \mathrm{e}-2$ & $2.25 \mathrm{e}-2$ & $5.26 \mathrm{e}-3$ & $5.51 \mathrm{e}-3$ & $1.64 \mathrm{e}-3$ & $1.65 \mathrm{e}-3$
\end{tabular}

Fig. 7.6: Values of $\lambda$ for the exponential fitting with $A \exp (-\lambda t)$ of the kinetic energy computed for time $t \in[2,5]$ with the meshes at refinement levels $\ell=2,3,4,5$ and $\Delta t=0.1,0.01$.

term in the mass balance equation $(2.3)$ :

$$
g(\mathbf{r})=\frac{1}{h^{2}}\left(\exp \left(-\frac{\|\mathbf{r}-\mathbf{a}\|^{2}}{h^{2}}\right)-\exp \left(-\frac{\|\mathbf{r}-\mathbf{b}\|^{2}}{h^{2}}\right)\right)
$$

where $\mathbf{a}=(-1.0,1.0, \sqrt{(7+\sqrt{19}) / 3})$ and $\mathbf{b}=(1.0,-1.0,-\sqrt{(7+\sqrt{19}) / 3})$ are located on the manifold. Note that $g$ consists of fluid source and sink, which approximate point source and sink for $h \rightarrow 0$.

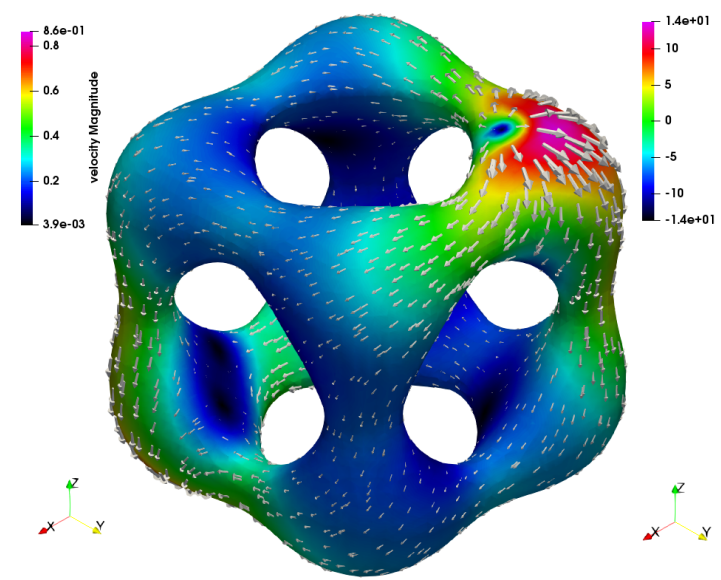

(a) Velocity

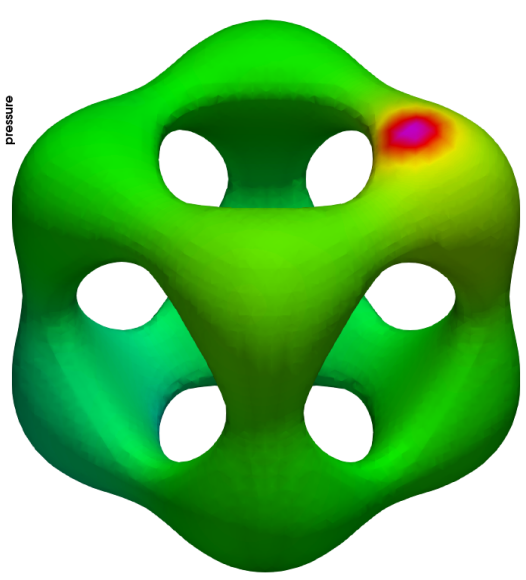

(b) Pressure

Fig. 7.7: Velocity and and pressure at $t=10$ computed with mesh $\ell=5$ and $\Delta t=0.1$.

We set mesh level $\ell=5$ and time step $\Delta t=0.1$. Figure 7.7 shows the computed velocity and pressure after they have (essentially) converged to an equilibrium state. The results were obtained for $c_{\tau}=10, c_{p}=1$, and $c_{u}=1$. This example illustrates the flexibility of the proposed numerical method in handling fluid problems over complex geometries without surface parametrization and mesh fitting.

\section{REFERENCES}

[1] M. Arnaudon And A. B. Cruzeiro, Lagrangian Navier-Stokes diffusions on manifolds: variational principle and stability, Bulletin des Sciences Mathématiques, 136 (2012), pp. 857881. 
[2] V. I. ARnol'D, Mathematical methods of classical mechanics, vol. 60, Springer Science \& Business Media, 2013.

[3] M. Arroyo And A. DeSimone, Relaxation dynamics of fluid membranes, Physical Review E, 79 (2009), p. 031915.

[4] J. W. Barrett, H. Garcke, And R. Nürnberg, A stable numerical method for the dynamics of fluidic membranes, Numerische Mathematik, 134 (2016), pp. 783-822.

[5] H. Brenner, Interfacial transport processes and rheology, Elsevier, 2013.

[6] F. Brezzi and J. Pitkäranta, On the Stabilization of Finite Element Approximations of the Stokes Equations, Vieweg+Teubner Verlag, Wiesbaden, 1984, pp. 11-19.

[7] E. Burman, P. Hansbo, M. G. Larson, and A. Massing, Cut finite element methods for partial differential equations on embedded manifolds of arbitrary codimensions, arXiv preprint arXiv:1610.01660, (2016).

[8] C. CaO, M. A. Rammaha, and E. S. Titi, The Navier-Stokes equations on the rotating 2-d sphere: Gevrey regularity and asymptotic degrees of freedom, Zeitschrift für angewandte Mathematik und Physik ZAMP, 50 (1999), pp. 341-360.

[9] A. Y. Chernyshenko And M. A. Olshanskit, An adaptive octree finite element method for PDEs posed on surfaces, Computer Methods in Applied Mechanics and Engineering, 291 (2015), pp. 146-172.

[10] A. Demlow And M. Olshanskil, An adaptive surface finite element method based on volume meshes, SIAM J. Numer. Anal., 50 (2012), pp. 1624-1647.

[11] G. Dziuk and C. M. Elliott, Finite element methods for surface PDEs, Acta Numerica, 22 (2013), pp. 289-396.

[12] D. G. Ebin And J. Marsden, Groups of diffeomorphisms and the motion of an incompressible fluid, Annals of Mathematics, (1970), pp. 102-163.

[13] H. Elman, D. Silvester, And A. Wathen, Finite elements and fast iterative solvers, Oxford University Press, Oxford, 2005.

[14] A. Ern and J.-L. Guermond, Theory and practice of finite elements, vol. 159, Springer Science \& Business Media, 2013.

[15] T.-P. FrIEs, Higher-order surface FEM for incompressible Navier-Stokes flows on manifolds, arXiv preprint arXiv:1712.02520, (2017).

[16] J. Grande, C. Lehrenfeld, And A. Reusken, Analysis of a high order trace finite element method for PDEs on level set surfaces, IMA Journal of Numerical Analysis, (2017). To appear.

[17] S. Gross, T. Jankuhn, M. A. Olshanskit, and A. Reusken, A trace finite element method for vector-Laplacians on surfaces, arXiv preprint arXiv:1709.00479 (to appear in SINUM), (2017).

[18] M. E. Gurtin And A. I. Murdoch, A continuum theory of elastic material surfaces, Archive for Rational Mechanics and Analysis, 57 (1975), pp. 291-323.

[19] J. Guzmán And M. OlshanskiI, Inf-sup stability of geometrically unfitted Stokes finite elements, Mathematics of Computation, (2017).

[20] A. Hansbo And P. Hansbo, An unfitted finite element method, based on Nitsche's method, for elliptic interface problems, Comput. Methods Appl. Mech. Engrg., 191 (2002), pp. 55375552.

[21] P. Hansbo and M. G. Larson, A stabilized finite element method for the Darcy problem on surfaces, IMA Journal of Numerical Analysis, (2016), p. drw041.

[22] P. Hansbo, M. G. Larson, and K. Larsson, Analysis of finite element methods for vector Laplacians on surfaces, arXiv preprint arXiv:1610.06747, (2016).

[23] M. Holst And A. Stern, Geometric variational crimes: Hilbert complexes, finite element exterior calculus, and problems on hypersurfaces., Foundations of Computational Mathematics, 12 (2012).

[24] T. Jankuhn, M. A. Olshanskit, And A. Reusken, Incompressible fluid problems on embedded surfaces: Modeling and variational formulations, Preprint arXiv:1702.02989, (2017).

[25] H. KobA, C. LiU, And Y. Giga, Energetic variational approaches for incompressible fluid systems on an evolving surface, Quarterly of Applied Mathematics, (2016).

[26] C. Lehrenfeld, M. A. Olshanskit, and X. Xu, A stabilized trace finite element method for partial differential equations on evolving surfaces, arXiv preprint arXiv:1709.07117, (2017).

[27] M. Mitrea And M. TAYlor, Navier-Stokes equations on Lipschitz domains in Riemannian manifolds, Mathematische Annalen, 321 (2001), pp. 955-987.

[28] I. Nitschke, A. Voigt, AND J. Wensch, A finite element approach to incompressible two-phase flow on manifolds, Journal of Fluid Mechanics, 708 (2012), pp. 418-438.

[29] M. Olshanski and A. Reusken, A finite element method for surface PDEs: matrix properties, Numer. Math., 114 (2009), pp. 491-520. 
[30] M. Olshanskit, A. Reusken, and J. Grande, A finite element method for elliptic equations on surfaces, SIAM J. Numer. Anal., 47 (2009), pp. 3339-3358.

[31] M. A. Olshanskit And A. Reusken, Trace finite element methods for PDEs on surfaces, arXiv preprint arXiv:1612.00054 (to appear in LNCSE V. 121), (2016).

[32] M. A. Olshanskit, A. Reusken, and X. Xu, An Eulerian space-time finite element method for diffusion problems on evolving surfaces, SIAM journal on numerical analysis, 52 (2014), pp. 1354-1377.

[33] M. Rahimi, A. DeSimone, and M. Arroyo, Curved fluid membranes behave laterally as effective viscoelastic media, Soft Matter, 9 (2013), pp. 11033-11045.

[34] P. Rangamani, A. Agrawal, K. K. Mandadapu, G. Oster, and D. J. Steigmann, Interaction between surface shape and intra-surface viscous flow on lipid membranes, Biomechanics and modeling in mechanobiology, (2013), pp. 1-13.

[35] A. Reusken, Analysis of trace finite element methods for surface partial differential equations, IMA Journal of Numerical Analysis, 35 (2015), pp. 1568-1590.

[36] A. Reusken And Y. Zhang, Numerical simulation of incompressible two-phase flows with a Boussinesq-Scriven surface stress tensor, Numerical Methods in Fluids, 73 (2013), pp. 1042-1058.

[37] S. Reuther AND A. Voigt, The interplay of curvature and vortices in flow on curved surfaces, Multiscale Modeling \& Simulation, 13 (2015), pp. 632-643.

[38] - Solving the incompressible surface navier-stokes equation by surface finite elements, arXiv preprint arXiv:1709.02803, (2017).

[39] T. SAKAI, Riemannian geometry, vol. 149, American Mathematical Soc., 1996.

[40] L. SCRIVen, Dynamics of a fluid interface equation of motion for Newtonian surface fluids, Chemical Engineering Science, 12 (1960), pp. 98-108.

[41] J. C. Slattery, L. Sagis, and E.-S. OH, Interfacial transport phenomena, Springer Science \& Business Media, 2007

[42] M. E. TAYLOR, Analysis on Morrey spaces and applications to Navier-Stokes and other evolution equations, Communications in Partial Differential Equations, 17 (1992), pp. 14071456.

[43] R. Temam, Infinite-dimensional dynamical systems in mechanics and physics, Springer, New York, 1988. 